\title{
A LOCAL DISCONTINUOUS GALERKIN METHOD FOR NONLINEAR PARABOLIC SPDES
}

\author{
Yunzhang Li $^{1}$, Chi-Wang Shu ${ }^{2, *}$ And Shanjian Tang ${ }^{1}$
}

\begin{abstract}
In this paper, we propose a local discontinuous Galerkin (LDG) method for nonlinear and possibly degenerate parabolic stochastic partial differential equations, which is a high-order numerical scheme. It extends the discontinuous Galerkin (DG) method for purely hyperbolic equations to parabolic equations and shares with the DG method its advantage and flexibility. We prove the $L^{2}$-stability of the numerical scheme for fully nonlinear equations. Optimal error estimates $\left(\mathcal{O}\left(h^{k+1}\right)\right)$ for smooth solutions of semi-linear stochastic equations is shown if polynomials of degree $k$ are used. We use an explicit derivative-free order 1.5 time discretization scheme to solve the matrix-valued stochastic ordinary differential equations derived from the spatial discretization. Numerical examples are given to display the performance of the LDG method.
\end{abstract}

Mathematics Subject Classification. 65C30, 60H35.

Received May 18, 2019. Accepted April 13, 2020.

\section{INTRODUCTION}

In this paper we present a local discontinuous Galerkin (LDG) method for nonlinear parabolic stochastic partial differential equations (SPDEs) with a periodic boundary condition and a multiplicative noise of the form:

$$
\left\{\begin{array}{cc}
\mathrm{d} u=\left\{\left[a\left(\cdot, x, t, u, u_{x}\right) u_{x}\right]_{x}+\psi\left(\cdot, x, t, u, u_{x}\right)\right\} \mathrm{d} t+g\left(\cdot, x, t, u, u_{x}\right) \mathrm{d} W_{t}, \\
(x, t) \in[0,2 \pi] \times(0, T] ; \\
u(x, 0)=u_{0}(x), & x \in[0,2 \pi],
\end{array}\right.
$$

where the terminal time $T>0$ is a fixed real number, $\left\{W_{t}, 0 \leq t \leq T\right\}$ is a standard one-dimensional Brownian motion on a given probability space $(\Omega, \mathcal{F}, \mathbb{P}),\left\{\mathcal{F}_{t}, 0 \leq t \leq T\right\}$ denotes its augmented natural filtration, and the real scalar-valued functions $a, \psi$ and $g$ are all $\mathcal{F} \otimes \mathcal{B}\left([0,2 \pi] \times[0, T] \times \mathbb{R}^{2}\right)$-measurable. Notice that the assumption of periodic boundary conditions is for simplicity of exposition only and is not essential: the method as well as the analysis can be easily adapted for non-periodic boundary conditions. We make the following hypotheses:

Keywords and phrases. Local discontinuous Galerkin method, nonlinear parabolic stochastic partial differential equations, multiplicative noise, stability analysis, error estimates, stochastic viscous Burgers equation.

1 Department of Finance and Control Sciences, School of Mathematical Sciences, Fudan University, Shanghai 200433,

P.R. China.

2 Division of Applied Mathematics, Brown University, Providence, RI 02912, USA.

*Corresponding author: Chi-Wang_Shu@brown.edu 
(H1) The initial condition $u_{0} \in L^{2}(0,2 \pi)$.

(H2) The leading coefficient $a$ is locally Lipschitz continuous in the last two variables. There exist two nonnegative constants $\alpha$ and $\Lambda$ such that

$$
\alpha \leq a(\omega, x, t, u, v) \leq \Lambda
$$

for any $(\omega, x, t, u, v) \in \Omega \times[0,2 \pi] \times[0, T] \times \mathbb{R}^{2}$.

(H3) There exist three positive constants $B_{1}, B_{2}$, and $B_{3}$ such that

$$
\left|\psi(\omega, x, t, u, v)-\psi\left(\omega, x, t, u^{\prime}, v^{\prime}\right)\right| \leq B_{1}\left(\left|u-u^{\prime}\right|+\left|v-v^{\prime}\right|\right)
$$

and

$$
|\psi(\omega, x, t, u, v)|^{2} \leq B_{2}^{2}\left(1+|u|^{2}\right)+B_{3}^{2}|v|^{2}
$$

for any $\left(\omega, x, t ; u, u^{\prime}, v, v^{\prime}\right) \in \Omega \times[0,2 \pi] \times[0, T] \times \mathbb{R}^{4}$.

(H4) There are four nonnegative constants $C_{i}$ with $i=1,2,3,4$ such that

$$
\left|g(\omega, x, t, u, v)-g\left(\omega, x, t, u^{\prime}, v^{\prime}\right)\right| \leq C_{1}\left|u-u^{\prime}\right|+C_{2}\left|v-v^{\prime}\right|
$$

and

$$
|g(\omega, x, t, u, v)|^{2} \leq C_{3}^{2}\left(1+|u|^{2}\right)+C_{4}^{2}|v|^{2}
$$

for any $\left(\omega, x, t ; u, u^{\prime}, v, v^{\prime}\right) \in \Omega \times[0,2 \pi] \times[0, T] \times \mathbb{R}^{4}$.

Various phenomena and applications (see $[36,39]$ and the references therein) with stochastic influence in natural or artificial complex systems can be modeled by SPDEs (1.1), including stochastic quantization of the free Euclidean quantum field, turbulence, population dynamics and genetics, neurophysiology, evolution of the curve of interest rate, nonlinear filtering, movement by mean curvature in random environment, hydrodynamic limit of particle systems, fluctuations of an interface on a wall, and pathwise stochastic control theory. In these fundamental applications, several examples of canonical SPDEs arise, such as the Zakai equation, reflected stochastic heat equation, stochastic reaction diffusion equations, stochastic Burgers equation, stochastic NavierStokes equation, and stochastic porous media equation.

Concerning the theoretical study for nonlinear SPDEs with a multiplicative stochastic forcing term involving a temporary white noise, Pardoux and Peng [37] proved existence and uniqueness of a classical solution by establishing the connection with backward doubly stochastic differential equations (BDSDEs). Hofmanová [24] obtained a regularity result for the strong solution with periodic boundary condition when all the coefficients are sufficiently smooth. Recently, Du and Liu [18] gave a Schauder estimate for linear SPDEs, which can be suitably generalized to nonlinear cases. In addition to these, there are also numerous research activities on nonlinear SPDEs. See e.g. $[3,15,20,43]$. However, in most cases it is not available to have explicit solutions to the SPDEs, and numerical solutions of SPDEs naturally receive a lot of attentions.

In recent years, numerous studies have been focused on advanced and efficient methods for SPDEs such as finite difference methods $[16,21,22,33,42,45]$, finite element methods $[1,17,19,28,44,47]$, spectral methods [25, $31,34,35]$, and also some other types of numerical methods [7,41]. Concerning discontinuous finite element methods for SPDEs, Cao et al. $[4,5]$ developed a discontinuous Galerkin (DG) method to the time-independent elliptic SPDEs with additive noises. Li et al. [30] proposed a DG method for nonlinear stochastic hyperbolic conservation laws, in which they investigated the stability for fully nonlinear equations and the error estimates for semilinear equations. Pazner et al. [38] formulated an LDG scheme on the basis of fluctuation-dissipation balance to approximate linear parabolic SPDEs driven by additive noises, which preserves a discrete fluctuationdissipation structure, but neither stability nor any error estimate is given. To the best of our knowledge, little attention has been paid to the stability and error estimates of high-order approximate schemes for fully nonlinear parabolic SPDEs with multiplicative noises.

The LDG method was introduced by Cockburn and Shu [12] as a generalization of the numerical scheme proposed by Bassi and Rebay [2] for the compressible Navier-Stokes equations. This scheme was in turn an 
extension of the DG method developed by Cockburn et al. [9-11,13,14] for nonlinear hyperbolic systems. With the help of the local Gauss-Radau projection, the $L^{2}$-norm stability and optimal error estimates are obtained for deterministic problems $[6,46]$, if the alternating numerical fluxes are used. In this paper, we shall consider stochastic counterparts of these works and propose an LDG scheme for the nonlinear parabolic SPDEs (1.1). Our numerical scheme shares the following advantages and flexibilities of the classical DG method: (1) it is easy to design high order approximations, thus allowing for efficient $p$-adaptivity; (2) it is flexible on complicated geometries, thus allowing for efficient $h$-adaptivity; $(3)$ it is local in data communications, thus allowing for efficient parallel implementations.

It should be pointed out that our effective computational methods for SPDEs have new difficulties. A solution of SPDEs, even when it exists, is not time-differentiable in nature, and is not bounded in general in the path. These new features complicate our calculation and analysis. Using the similar techniques for stochastic hyperbolic equations with DG methods in [30], we properly estimate the quadratic covariation process of the approximating solution to obtain stability and optimal error estimates.

As an extension of the DG method, the LDG method can not only deal with nonlinear multiplicative noise containing the unknown variable $u$ itself, but also nicely handle the case of its first order spatial derivative $u_{x}$ being involved in the stochastic diffusion term $g$, as long as the stochastic parabolicity $2 \alpha \geq C_{4}^{2}$ is satisfied. Though few studies are given on unique solvability and regularity of strong solutions to degenerate nonlinear SPDEs, we can design the LDG scheme for the degenerate case $2 \alpha=C_{4}^{2}$ and prove its stability, which is confirmed by numerical tests for degenerate SPDEs in Section 7. These numerical experiments further indicate that our scheme also has optimal order of accuracy even in the degenerate case.

Our high-order approximation scheme can be more efficient for high-accuracy computation of the smooth case, which is rather attractive in applications. However, for the discontinuous case, our scheme loses the high order of accuracy and has spurious numerical oscillations near discontinuous region. In practice, it is worth trying to use limiters to control oscillations for the discontinuous problems, which remain to be investigated in the future. Our numerical algorithm and stability analysis are restricted within the one-dimensional spacial case, but they can be generalized to higher spacial dimensions in a straightforward way. The optimal error estimate will however be more involved in the multi-dimensional spacial case, especially on unstructured meshes, which remains to be studied in the future.

The paper is organized as follows. In Section 2, we introduce notations, definitions and auxiliary results used in the paper. In Section 3, we present the LDG method for nonlinear parabolic SPDEs (1.1), and study the existence and uniqueness of the solution to the stochastic differential equations (SDEs) derived from the spatial discretization. In Section 4 , we investigate the $L^{2}$-stability for the fully nonlinear stochastic equations. In section 5 , we obtain the $L^{2}$-norm optimal error estimates $\left(\mathcal{O}\left(h^{k+1}\right)\right)$ for semilinear stochastic equations. In Section 6 , we use a derivative-free order 1.5 scheme for matrix-valued SDEs as time discretization, to collaborate with the semi-discrete LDG scheme. Finally the paper ends with a series of numerical experiments on some model problems in Section 7, which confirm the analytical results.

\section{NOtATIONS, DEFINITIONS AND AUXILIARY RESUltS}

In this section, we introduce notations, definitions, and some auxiliary results.

\subsection{Notations}

We denote the mesh by $I_{j}=\left[x_{j-\frac{1}{2}}, x_{j+\frac{1}{2}}\right]$, for $j=1, \ldots, N$. The nodes are denoted by $\left\{x_{j+\frac{1}{2}}, j=\right.$ $0,1, \ldots, N\}$ with $x_{\frac{1}{2}}=0$ and $x_{N+\frac{1}{2}}=2 \pi$. The mesh size is denoted by $h_{j}=x_{j+\frac{1}{2}}-x_{j-\frac{1}{2}}$, with $h=\max _{1 \leq j \leq N} h_{j}$ being the maximum mesh size. We assume that the mesh is regular, namely the ratio between the maximum and the minimum mesh sizes stays bounded during mesh refinements. We define the piecewise polynomial space $V_{h}$ as the space of polynomials of the degree up to $k$ in each cell $I_{j}$, i.e.

$$
V_{h}=\left\{v \mid v \in P^{k}\left(I_{j}\right) \text { for } x \in I_{j}, j=1, \ldots, N\right\} .
$$


Note that functions in $V_{h}$ might have discontinuities on an element interface.

We consider the Sobolev space $\|\cdot\|_{H^{m, p}}$ with periodic boundary condition,

$$
\begin{aligned}
H^{m, p}:=\left\{u:[0,2 \pi] \rightarrow \mathbb{R} \mid\|u\|_{H^{m, p}}\right. & =\left[\int_{0}^{2 \pi}\left(|u(x)|^{p}+\sum_{l=1}^{m}\left|\frac{\mathrm{d}^{l}}{\mathrm{~d} x^{l}} u(x)\right|^{p}\right) \mathrm{d} x\right]^{\frac{1}{p}}<\infty, \\
u(0) & \left.=u(2 \pi), \frac{\mathrm{d}^{l}}{\mathrm{~d} x^{l}} u(0)=\frac{\mathrm{d}^{l}}{\mathrm{~d} x^{l}} u(2 \pi), l=1, \ldots, m-1\right\} .
\end{aligned}
$$

For simplicity, we write $\|\cdot\|_{H^{m}}$ for $\|\cdot\|_{H^{m, 2}}$, and $\|\cdot\|$ for the $L^{2}(0,2 \pi)$ norm. We denote by $\mathcal{S}^{2}\left(\Omega \times[0, T] ; L^{2}\right)$, the space of all adapted strongly continuous processes $\phi: \Omega \times[0, T] \longrightarrow L^{2}(0,2 \pi)$ such that

$$
\|\phi\|_{\mathcal{S}^{2}\left(\Omega \times[0, T] ; L^{2}\right)}:=\left(\mathbb{E}\left[\sup _{0 \leq t \leq T}\|\phi(t)\|^{2}\right]\right)^{\frac{1}{2}}<\infty .
$$

An element of $\mathbb{R}^{n \times d}$ is a $n \times d$ matrix, and its Euclidean norm is given by $|y|:=\sqrt{\operatorname{trace}\left(y y^{*}\right)}$ for $y \in \mathbb{R}^{n \times d}$.

The solution of the numerical scheme is denoted by $u_{h}$, and belongs to the finite element space $V_{h}$. We denote by $u_{j+\frac{1}{2}}^{+}$and $u_{j+\frac{1}{2}}^{-}$the right and left limits of the function $u$ at $x_{j+\frac{1}{2}}$, respectively.

By $\stackrel{C}{C}>0$, we denote a generic constant, which in particular does not depend on the discretization width $h$ and possibly changes from line to line. Since the Itô integral is not defined in a pathwise sense, the argument $\omega$ of the integrand as a stochastic process will be omitted in the rest of this paper if there is no danger of confusion.

\subsection{Projection properties}

We consider the standard $L^{2}$-projection (denoted by $\mathcal{P}$ ), and the local Gauss-Radau projections $\mathcal{R}$ and $\mathcal{Q}$ into space $V_{h}$. For each $j$, the projections satisfy that

$$
\begin{gathered}
\int_{I_{j}}[\mathcal{P} u(x)-u(x)] v(x) \mathrm{d} x=0, \quad \forall v \in P^{k}\left(I_{j}\right), \\
\int_{I_{j}}[\mathcal{R} u(x)-u(x)] v(x) \mathrm{d} x=0, \quad \forall v \in P^{k-1}\left(I_{j}\right), \quad \text { and } \quad \mathcal{R} u\left(x_{j-\frac{1}{2}}^{+}\right)=u\left(x_{j-\frac{1}{2}}\right),
\end{gathered}
$$

and

$$
\int_{I_{j}}[\mathcal{Q} u(x)-u(x)] v(x) \mathrm{d} x=0, \quad \forall v \in P^{k-1}\left(I_{j}\right), \quad \text { and } \quad \mathcal{Q} u\left(x_{j+\frac{1}{2}}^{-}\right)=u\left(x_{j+\frac{1}{2}}\right) .
$$

Furthermore, we have (c.f. [8])

$$
\|\mathcal{P} u-u\|+\|\mathcal{R} u-u\|+\|\mathcal{Q} u-u\| \leq C\|u\|_{H^{k+1}} h^{k+1},
$$

where the positive constant $C$ is independent of $u$ and $h$.

\subsection{Properties of the Itô formula}

For continuous semi-martingales $X$ and $Y$, we have

$$
X_{t} Y_{t}=X_{0} Y_{0}+\int_{0}^{t} X_{s} \mathrm{~d} Y_{s}+\int_{0}^{t} Y_{s} \mathrm{~d} X_{s}+\langle X, Y\rangle_{t}
$$

where $\langle X, Y\rangle$ is the quadratic covariation process of $X$ and $Y$. Note that $\langle X, Y\rangle=\langle Y, X\rangle$. For any locally bounded adapted process $H$, we have

$$
\left\langle\int_{0}^{\cdot} H_{s} \mathrm{~d} X_{s}, Y\right\rangle_{t}=\int_{0}^{t} H_{s} \mathrm{~d}\langle X, Y\rangle_{s}
$$


Moreover, if $X$ is continuous and is of bounded total variation, we have

$$
\langle X, Y\rangle=0 .
$$

The following lemma is well-known in the martingale theory. See e.g. [23], Theorem 10.19, page 273.

Lemma 2.1. If $\mathbb{E}\left[\left(\int_{0}^{T} H_{s}^{2} \mathrm{~d} s\right)^{\frac{1}{2}}\right]<\infty$, then $\left\{\int_{0}^{t} H_{s} \mathrm{~d} W_{s}, 0 \leq t \leq T\right\}$ is a martingale.

For more details on the Itô formula, the reader is referred to $[23,40]$.

\section{The LDG MEthod FOR NONLINEAR PARABOlic SPDES}

\subsection{The semi-discrete LDG method}

In this subsection, we formulate the LDG method for fully nonlinear parabolic SPDEs. As a special class of the DG methods, the main technique of the method is to rewrite (1.1) into an equivalent system containing only first-order spatial derivatives, which is further discretized by the standard DG method with correct definition of numerical fluxes. To do this, firstly, we rewrite the problem as a first-order system:

$$
\begin{cases}\mathrm{d} u=\left[w_{x}+\psi(\cdot, x, t, u, v)\right] \mathrm{d} t+g(\cdot, x, t, u, v) \mathrm{d} W_{t}, & (x, t) \in[0,2 \pi] \times(0, T] \\ v(x, t)=u_{x}(x, t), & (x, t) \in[0,2 \pi] \times(0, T] \\ w(x, t)=a(\cdot, x, t, u(x, t), v(x, t)) v(x, t), & (x, t) \in[0,2 \pi] \times(0, T] \\ u(x, 0)=u_{0}(x), & x \in[0,2 \pi] .\end{cases}
$$

The LDG method for (1.1) is now obtained by simply discretizing the above system with the DG method. We seek an approximation $\left(u_{h}, v_{h}, w_{h}\right)^{T}$ to the exact solution $(u, v, w)^{T}$ such that for any $(\omega, t) \in \Omega \times[0, T]$, $u_{h}(\omega, \cdot, t), v_{h}(\omega, \cdot, t)$ and $w_{h}(\omega, \cdot, t)$ belong to the finite dimensional space $V_{h}$. In order to determine the approximate solution $\left(u_{h}, v_{h}, w_{h}\right)^{T}$, we first note that by multiplying (3.1a), (3.1b), (3.1c), and (3.1d) with arbitrary smooth functions $r, z, p$ and $q$, respectively, and integrating over $I_{j}$ with $j=1,2, \ldots, N$, we get, after a simple formal integration by parts in (3.1a) and (3.1b),

$$
\begin{aligned}
\int_{I_{j}} r(x) \mathrm{d} u(\omega, x, t) \mathrm{d} x= & \left\{-\int_{I_{j}} w(\omega, x, t) r_{x}(x) \mathrm{d} x\right. \\
& +w\left(\omega, x_{j+\frac{1}{2}}, t\right) r\left(x_{j+\frac{1}{2}}^{-}\right)-w\left(\omega, x_{j-\frac{1}{2}}, t\right) r\left(x_{j-\frac{1}{2}}^{+}\right) \\
& \left.+\int_{I_{j}} \psi(\omega, x, t, u(\omega, x, t), v(\omega, x, t)) r(x) \mathrm{d} x\right\} \mathrm{d} t \\
& +\int_{I_{j}} g(\omega, x, t, u(\omega, x, t), v(\omega, x, t)) r(x) \mathrm{d} x \mathrm{~d} W_{t}, \\
\int_{I_{j}} v(\omega, x, t) z(x) \mathrm{d} x= & -\int_{I_{j}} u(\omega, x, t) z_{x}(x) \mathrm{d} x \\
& +u\left(\omega, x_{j+\frac{1}{2}}, t\right) z\left(x_{j+\frac{1}{2}}^{-}\right)-u\left(\omega, x_{j-\frac{1}{2}}, t\right) z\left(x_{j-\frac{1}{2}}^{+}\right), \\
\int_{I_{j}} w(\omega, x, t) p(x) \mathrm{d} x= & \int_{I_{j}} a(\omega, x, t, u(\omega, x, t), v(\omega, x, t)) v(\omega, x, t) p(x) \mathrm{d} x, \\
\int_{I_{j}} u(\omega, x, 0) q(x) \mathrm{d} x= & \int_{I_{j}} u_{0}(x) q(x) \mathrm{d} x .
\end{aligned}
$$


Next, we replace the smooth functions $r, z, p$ and $q$ with test functions $r_{h}, z_{h}, p_{h}$ and $q_{h}$, respectively, in the finite element space $V_{h}$ and the exact solution $(u, v, w)^{T}$ with the approximation $\left(u_{h}, v_{h}, w_{h}\right)^{T}$. Since the functions in $V_{h}$ might have discontinuities on an element interface, we must also replace the boundary terms

$$
w\left(\omega, x_{j+\frac{1}{2}}, t\right), \quad u\left(\omega, x_{j+\frac{1}{2}}, t\right)
$$

with the numerical fluxes

$$
\widehat{w}_{j+\frac{1}{2}}(\omega, t), \quad \widehat{u}_{j+\frac{1}{2}}(\omega, t)
$$

respectively, which will be suitably chosen later. Thus, the approximate solution given by the LDG method is defined as the solution of the following weak formulation:

$$
\begin{aligned}
\int_{I_{j}} r_{h}(x) \mathrm{d} u_{h}(\omega, x, t) \mathrm{d} x= & \left\{-\int_{I_{j}} w_{h}(\omega, x, t) r_{h x}(x) \mathrm{d} x\right. \\
& +\widehat{w}_{j+\frac{1}{2}}(\omega, t) r_{h}\left(x_{j+\frac{1}{2}}^{-}\right)-\widehat{w}_{j-\frac{1}{2}}(\omega, t) r_{h}\left(x_{j-\frac{1}{2}}^{+}\right) \\
& \left.+\int_{I_{j}} \psi\left(\omega, x, t, u_{h}(\omega, x, t), v_{h}(\omega, x, t)\right) r_{h}(x) \mathrm{d} x\right\} \mathrm{d} t \\
& +\int_{I_{j}} g\left(\omega, x, t, u_{h}(\omega, x, t), v_{h}(\omega, x, t)\right) r_{h}(x) \mathrm{d} x \mathrm{~d} W_{t}, \\
\int_{I_{j}} v_{h}(\omega, x, t) z_{h}(x) \mathrm{d} x= & -\int_{I_{j}} u_{h}(\omega, x, t) z_{h x}(x) \mathrm{d} x \\
& +\widehat{u}_{j+\frac{1}{2}}(\omega, t) z_{h}\left(x_{j+\frac{1}{2}}^{-}\right)-\widehat{u}_{j-\frac{1}{2}}(\omega, t) z_{h}\left(x_{j-\frac{1}{2}}^{+}\right), \\
\int_{I_{j}} w_{h}(\omega, x, t) p_{h}(x) \mathrm{d} x= & \int_{I_{j}}\left[a\left(\cdot, u_{h}, v_{h}\right) v_{h}\right](\omega, x, t) p_{h}(x) \mathrm{d} x, \\
\int_{I_{j}} u_{h}(\omega, x, 0) q_{h}(x) \mathrm{d} x= & \int_{I_{j}} u_{0}(x) q_{h}(x) \mathrm{d} x,
\end{aligned}
$$

for any $\left(r_{h}, z_{h}, p_{h}, q_{h}\right) \in\left(V_{h}\right)^{4}$. It only remains to choose suitable numerical fluxes. For $j=0,1, \ldots, N$, we choose

$$
\widehat{w}_{j+\frac{1}{2}}(\omega, t):=w_{h}\left(\omega, x_{j+\frac{1}{2}}^{+}, t\right), \quad \widehat{u}_{j+\frac{1}{2}}(\omega, t):=u_{h}\left(\omega, x_{j+\frac{1}{2}}^{-}, t\right) .
$$

Note that, by periodicity, we have

$$
\widehat{w}_{N+\frac{1}{2}}=\widehat{w}_{\frac{1}{2}}, \quad \widehat{u}_{\frac{1}{2}}=\widehat{u}_{N+\frac{1}{2}} .
$$

Remark 3.1. The choice of $(\widehat{w}, \widehat{u})$ in $(3.3)$ is referred to as the alternating flux, which is essential for the proof of optimal error estimates. We can also define the numerical flux in an alternating way as follows:

$$
\widehat{w}_{j+\frac{1}{2}}(\omega, t):=w_{h}\left(\omega, x_{j+\frac{1}{2}}^{-}, t\right), \quad \widehat{u}_{j+\frac{1}{2}}(\omega, t):=u_{h}\left(\omega, x_{j+\frac{1}{2}}^{+}, t\right) .
$$

\subsection{The stochastic ordinary differential equation derived from the spatial discretization}

The LDG method, as a spatial discretization, transfers the primal problem into a system of ordinary stochastic differential equations, which will be specified in this subsection. For $x \in I_{j}$, the numerical solution should have the form

$$
u_{h}(\omega, x, t)=\sum_{l=0}^{k} \mathbf{u}_{l, j}(\omega, t) \varphi_{l}^{j}(x), \quad v_{h}(\omega, x, t)=\sum_{l=0}^{k} \mathbf{v}_{l, j}(\omega, t) \varphi_{l}^{j}(x),
$$


and

$$
w_{h}(\omega, x, t)=\sum_{l=0}^{k} \mathbf{w}_{l, j}(\omega, t) \varphi_{l}^{j}(x),
$$

where $\left\{\varphi_{l}^{j}, l=0,1, \ldots, k\right\}$ is an arbitrary basis of $P^{k}\left(I_{j}\right)$.

By periodicity, we define the "ghost" coefficients as follows:

$$
\begin{array}{cl}
\mathbf{u}_{l, 0}=\mathbf{u}_{l, N}, & \mathbf{v}_{l, 0}=\mathbf{v}_{l, N}, \quad \mathbf{w}_{l, 0}=\mathbf{w}_{l, N}, \\
\mathbf{u}_{l, N+1}=\mathbf{u}_{l, 1}, & \mathbf{v}_{l, N+1}=\mathbf{v}_{l, 1}, \quad \mathbf{w}_{l, N+1}=\mathbf{w}_{l, 1} .
\end{array}
$$

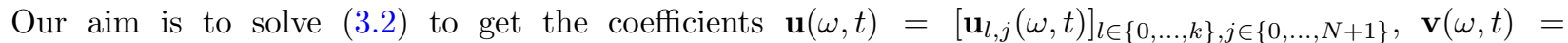
$\left[\mathbf{v}_{l, j}(\omega, t)\right]_{l \in\{0, \ldots, k\}, j \in\{0, \ldots, N+1\}}$ and $\mathbf{w}(\omega, t)=\left[\mathbf{w}_{l, j}(\omega, t)\right]_{l \in\{0, \ldots, k\}, j \in\{0, \ldots, N+1\}}$.

\subsubsection{Representation for $\mathbf{v}(\omega, t)$}

For $j=1,2, \ldots, N$, by taking $z_{h}:=\varphi_{m}^{j}$ for $m=0,1, \ldots, k$ in equality (3.2b), we have

$$
\begin{aligned}
\sum_{l=0}^{k}\left(\int_{I_{j}} \varphi_{m}^{j}(x) \varphi_{l}^{j}(x) \mathrm{d} x\right) \mathbf{v}_{l, j}(\omega, t)= & -\int_{I_{j}} \sum_{n=0}^{k} \mathbf{u}_{n, j}(\omega, t) \varphi_{n}^{j}(x) \varphi_{m x}^{j}(x) \mathrm{d} x \\
& +\sum_{n=0}^{k} \mathbf{u}_{n, j}(\omega, t) \varphi_{n}^{j}\left(x_{j+\frac{1}{2}}\right) \varphi_{m}^{j}\left(x_{j+\frac{1}{2}}\right) \\
& -\sum_{n=0}^{k} \mathbf{u}_{n, j-1}(\omega, t) \varphi_{n}^{j-1}\left(x_{j-\frac{1}{2}}\right) \varphi_{m}^{j}\left(x_{j-\frac{1}{2}}\right) .
\end{aligned}
$$

The mass matrix $A^{j}:=\left[A_{m l}^{j}\right]$ with

$$
A_{m l}^{j}:=\int_{I_{j}} \varphi_{m}^{j}(x) \varphi_{l}^{j}(x) \mathrm{d} x
$$

is invertible, and its inverse is denoted by $A^{j,-1}$.

It gives that

$$
\mathbf{v}_{l, j}(\omega, t)=\mathbf{V}_{l, j}(\mathbf{u}(\omega, t)),
$$

where

$$
\begin{aligned}
\mathbf{V}_{l, j}(\mathbf{u}):= & -\int_{I_{j}} \sum_{n=0}^{k} \mathbf{u}_{n, j} \varphi_{n}^{j}(x) \sum_{m=0}^{k} A_{l m}^{j,-1} \varphi_{m x}^{j}(x) \mathrm{d} x \\
& +\sum_{n=0}^{k} \mathbf{u}_{n, j} \varphi_{n}^{j}\left(x_{j+\frac{1}{2}}\right) \sum_{m=0}^{k} A_{l m}^{j,-1} \varphi_{m}^{j}\left(x_{j+\frac{1}{2}}\right) \\
& -\sum_{n=0}^{k} \mathbf{u}_{n, j-1} \varphi_{n}^{j-1}\left(x_{j-\frac{1}{2}}\right) \sum_{m=0}^{k} A_{l m}^{j,-1} \varphi_{m}^{j}\left(x_{j-\frac{1}{2}}\right) .
\end{aligned}
$$

By periodicity, we have

$$
\mathbf{V}_{l, 0}=\mathbf{V}_{l, N}, \quad \mathbf{V}_{l, N+1}=\mathbf{V}_{l, 1}
$$




\subsubsection{Representation for $\mathbf{w}(\omega, t)$}

For $j=1,2, \ldots, N$, by taking $p_{h}:=\varphi_{m}^{j}$ for $m=0,1, \ldots, k$ in equality (3.2c), we have

$$
\begin{aligned}
\sum_{l=0}^{k}\left(\int_{I_{j}} \varphi_{m}^{j}(x) \varphi_{l}^{j}(x) \mathrm{d} x\right) \mathbf{w}_{l, j}(\omega, t)= & \int_{I_{j}} a\left(\omega, x, t, \sum_{n=0}^{k} \mathbf{u}_{n, j}(\omega, t) \varphi_{n}^{j}(x), \sum_{n=0}^{k} \mathbf{v}_{n, j}(\omega, t) \varphi_{n}^{j}(x)\right) \\
& \times \sum_{n=0}^{k} \mathbf{v}_{n, j}(\omega, t) \varphi_{n}^{j}(x) \varphi_{m}^{j}(x) \mathrm{d} x .
\end{aligned}
$$

It turns out that

$$
\mathbf{w}_{l, j}(\omega, t)=\mathbf{W}_{l, j}(\omega, t, \mathbf{u}(\omega, t))
$$

where

$$
\begin{aligned}
\mathbf{W}_{l, j}(\omega, t, \mathbf{u}):= & \int_{I_{j}}\left[a\left(\omega, x, t, \sum_{n=0}^{k} \mathbf{u}_{n, j} \varphi_{n}^{j}(x), \sum_{n=0}^{k} \mathbf{V}_{n, j}(\mathbf{u}) \varphi_{n}^{j}(x)\right)\right. \\
& \left.\times \sum_{n=0}^{k} \mathbf{V}_{n, j}(\mathbf{u}) \varphi_{n}^{j}(x) \sum_{m=0}^{k} A_{l m}^{j,-1} \varphi_{m}^{j}(x)\right] \mathrm{d} x .
\end{aligned}
$$

By periodicity, we have

$$
\mathbf{W}_{l, 0}=\mathbf{W}_{l, N}, \quad \mathbf{W}_{l, N+1}=\mathbf{W}_{l, 1}
$$

\subsubsection{Representation for $\mathbf{u}(\omega, t)$}

For $j=1,2, \ldots, N$, by taking $r_{h}:=\varphi_{m}^{j}$ for $m=0,1, \ldots, k$ in equality (3.2a), we have

$$
\begin{aligned}
\sum_{l=0}^{k}\left(\int_{I_{j}} \varphi_{m}^{j}(x) \varphi_{l}^{j}(x) \mathrm{d} x\right) d \mathbf{u}_{l, j}(\omega, t)= & -\int_{I_{j}} \sum_{n=0}^{k} \mathbf{w}_{n, j}(\omega, t) \varphi_{n}^{j}(x) \varphi_{m x}^{j}(x) \mathrm{d} x \mathrm{~d} t \\
& +\sum_{n=0}^{k}\left[\mathbf{w}_{n, j+1}(\omega, t) \varphi_{n}^{j+1}\left(x_{j+\frac{1}{2}}\right) \varphi_{m}^{j}\left(x_{j+\frac{1}{2}}\right)\right. \\
& \left.-\mathbf{w}_{n, j}(\omega, t) \varphi_{n}^{j}\left(x_{j-\frac{1}{2}}\right) \varphi_{m}^{j}\left(x_{j-\frac{1}{2}}\right)\right] \mathrm{d} t \\
& +\int_{I_{j}} \psi\left(\omega, x, t, \sum_{n=0}^{k} \mathbf{u}_{n, j}(\omega, t) \varphi_{n}^{j}(x), \sum_{n=0}^{k} \mathbf{v}_{n, j}(\omega, t) \varphi_{n}^{j}(x)\right) \varphi_{m}^{j}(x) \mathrm{d} x \mathrm{~d} t \\
& +\int_{I_{j}} g\left(\omega, x, t, \sum_{n=0}^{k} \mathbf{u}_{n, j}(\omega, t) \varphi_{n}^{j}(x), \sum_{n=0}^{k} \mathbf{v}_{n, j}(\omega, t) \varphi_{n}^{j}(x)\right) \varphi_{m}^{j}(x) \mathrm{d} x \mathrm{~d} W_{t} .
\end{aligned}
$$

Then we obtain the following SDE of $\mathbf{u}$ :

$$
d \mathbf{u}(t)=F(\cdot, t, \mathbf{u}(t)) \mathrm{d} t+G(\cdot, t, \mathbf{u}(t)) \mathrm{d} W_{t},
$$


where for $j=1,2, \ldots, N$ and $l=0,1, \ldots, k$,

$$
\begin{aligned}
F_{l, j}(\omega, t, \mathbf{u}):= & \int_{I_{j}} \psi\left(\omega, x, t, \sum_{n=0}^{k} \mathbf{u}_{n, j} \varphi_{n}^{j}(x), \sum_{n=0}^{k} \mathbf{V}_{n, j}(\mathbf{u}) \varphi_{n}^{j}(x)\right) \sum_{m=0}^{k} A_{l m}^{j,-1} \varphi_{m}^{j}(x) \mathrm{d} x \\
& -\int_{I_{j}} \sum_{n=0}^{k} \mathbf{W}_{n, j}(\omega, t, \mathbf{u}) \varphi_{n}^{j}(x) \sum_{m=0}^{k} A_{l m}^{j,-1} \varphi_{m x}^{j}(x) \mathrm{d} x \\
& +\sum_{n=0}^{k} \mathbf{W}_{n, j+1}(\omega, t, \mathbf{u}) \varphi_{n}^{j+1}\left(x_{j+\frac{1}{2}}\right) \sum_{m=0}^{k} A_{l m}^{j,-1} \varphi_{m}^{j}\left(x_{j+\frac{1}{2}}\right) \\
& -\sum_{n=0}^{k} \mathbf{W}_{n, j}(\omega, t, \mathbf{u}) \varphi_{n}^{j}\left(x_{j-\frac{1}{2}}\right) \sum_{m=0}^{k} A_{l m}^{j,-1} \varphi_{m}^{j}\left(x_{j-\frac{1}{2}}\right)
\end{aligned}
$$

and

$$
G_{l, j}(\omega, t, \mathbf{u}):=\int_{I_{j}} g\left(\omega, x, t, \sum_{n=0}^{k} \mathbf{u}_{n, j} \varphi_{n}^{j}(x), \sum_{n=0}^{k} \mathbf{V}_{n, j}(\mathbf{u}) \varphi_{n}^{j}(x)\right) \sum_{m=0}^{k} A_{l m}^{j,-1} \varphi_{m}^{j}(x) \mathrm{d} x,
$$

with periodic settings $F_{l, 0}=F_{l, N}, F_{l, N+1}=F_{l, 1}, G_{l, 0}=G_{l, N}$, and $G_{l, N+1}=G_{l, 1}$.

Lemma 3.2. Let assumptions (H2)-(H4) hold. Then for any $N \in \mathbb{N}_{+}$, the functions $F$ and $G$ are locally Lipschitz continuous in the variable $\mathbf{u}$, i.e., for any $M \in \mathbb{N}_{+}$, there is a positive constant $L_{N}(M)$ such that

$$
\left|F(\omega, t, \mathbf{u})-F\left(\omega, t, \mathbf{u}^{\prime}\right)\right|+\left|G(\omega, t, \mathbf{u})-G\left(\omega, t, \mathbf{u}^{\prime}\right)\right| \leq L_{N}(M)\left|\mathbf{u}-\mathbf{u}^{\prime}\right|
$$

for all $(\omega, t) \in \Omega \times[0, T]$ and all $\mathbf{u}, \mathbf{u}^{\prime} \in \mathbb{R}^{(k+1) \times(N+2)}$ with $|\mathbf{u}| \vee\left|\mathbf{u}^{\prime}\right| \leq M$ (where $a \vee b=\max (a, b)$ ).

Proof. We only show the local Lipschitz continuity of $F$ for fixed $N \in \mathbb{N}$, and that of $G$ can be proved in a similar way. The proof consists of the following three steps:

Step 1. We first show the uniform Lipschitz continuity of $\mathbf{V}$ for fixed $N \in \mathbb{N}$. For any $\mathbf{u}, \mathbf{u}^{\prime} \in \mathbb{R}^{(k+1) \times(N+2)}$, $l=0,1, \ldots, k$, and $j=1,2, \ldots, N$, we have

$$
\mathbf{V}_{l, j}(\mathbf{u})-\mathbf{V}_{l, j}\left(\mathbf{u}^{\prime}\right)=E_{1}^{l, j}+E_{2}^{l, j}+E_{3}^{l, j},
$$

where

$$
\begin{aligned}
& E_{1}^{l, j}:=-\int_{I_{j}} \sum_{n=0}^{k}\left(\mathbf{u}_{n, j}-\mathbf{u}_{n, j}^{\prime}\right) \varphi_{n}^{j}(x) \sum_{m=0}^{k} A_{l m}^{j,-1} \varphi_{m x}^{j}(x) \mathrm{d} x, \\
& E_{2}^{l, j}:=\sum_{n=0}^{k}\left(\mathbf{u}_{n, j}-\mathbf{u}_{n, j}^{\prime}\right) \varphi_{n}^{j}\left(x_{j+\frac{1}{2}}\right) \sum_{m=0}^{k} A_{l m}^{j,-1} \varphi_{m}^{j}\left(x_{j+\frac{1}{2}}\right), \\
& E_{3}^{l, j}:=-\sum_{n=0}^{k}\left(\mathbf{u}_{n, j-1}-\mathbf{u}_{n, j-1}^{\prime}\right) \varphi_{n}^{j-1}\left(x_{j-\frac{1}{2}}\right) \sum_{m=0}^{k} A_{l m}^{j,-1} \varphi_{m}^{j}\left(x_{j-\frac{1}{2}}\right) .
\end{aligned}
$$

Then we have

$$
\begin{aligned}
\left|E_{1}^{l, j}\right| & \leq \sum_{n=0}^{k} \int_{I_{j}}\left|\varphi_{n}^{j}(x)\right| \sum_{m=0}^{k}\left|\varphi_{m x}^{j}(x)\right| \mathrm{d} x\left\|A^{j,-1}\right\|_{\infty}\left|\mathbf{u}_{n, j}-\mathbf{u}_{n, j}^{\prime}\right| \\
& \leq \sum_{n=0}^{k} C_{N}\left|\mathbf{u}_{n, j}-\mathbf{u}_{n, j}^{\prime}\right| \leq C_{N}\left(\sum_{n=0}^{k}\left|\mathbf{u}_{n, j}-\mathbf{u}_{n, j}^{\prime}\right|^{2}\right)^{\frac{1}{2}},
\end{aligned}
$$


where $C_{N}$ is a positive constant which depends on $N$. Next, we have

$$
\begin{aligned}
\left|E_{2}^{l, j}\right| & \leq \sum_{n=0}^{k}\left|\varphi_{n}^{j}\left(x_{j+\frac{1}{2}}\right)\right|\left\|A^{j,-1}\right\|_{\infty} \sum_{m=0}^{k}\left|\varphi_{m}^{j}\left(x_{j+\frac{1}{2}}\right)\right|\left|\mathbf{u}_{n, j}-\mathbf{u}_{n, j}^{\prime}\right| \\
& \leq \sum_{n=0}^{k} C_{N}\left|\mathbf{u}_{n, j}-\mathbf{u}_{n, j}^{\prime}\right| \leq C_{N}\left(\sum_{n=0}^{k}\left|\mathbf{u}_{n, j}-\mathbf{u}_{n, j}^{\prime}\right|^{2}\right)^{\frac{1}{2}} .
\end{aligned}
$$

By similar calculation, we get that

$$
\left|E_{3}^{l, j}\right| \leq C_{N}\left(\sum_{n=0}^{k}\left|\mathbf{u}_{n, j-1}-\mathbf{u}_{n, j-1}^{\prime}\right|^{2}\right)^{\frac{1}{2}}
$$

It turns out that for any $l=0,1, \ldots, k, j=1,2, \ldots, N$

$$
\begin{aligned}
\left|\mathbf{V}_{l, j}(\mathbf{u})-\mathbf{V}_{l, j}\left(\mathbf{u}^{\prime}\right)\right|^{2} & \leq 3\left(\left|E_{1}^{l, j}\right|^{2}+\left|E_{2}^{l, j}\right|^{2}+\left|E_{3}^{l, j}\right|^{2}\right) \\
& \leq C_{N} \sum_{n=0}^{k}\left(\left|\mathbf{u}_{n, j}-\mathbf{u}_{n, j}^{\prime}\right|^{2}+\left|\mathbf{u}_{n, j-1}-\mathbf{u}_{n, j-1}^{\prime}\right|^{2}\right) .
\end{aligned}
$$

Then by the periodicity, it holds that

$$
\begin{aligned}
\left|\mathbf{V}(\mathbf{u})-\mathbf{V}\left(\mathbf{u}^{\prime}\right)\right|^{2} & =\sum_{l=0}^{k} \sum_{j=0}^{N+1}\left|\mathbf{V}_{l, j}(\mathbf{u})-\mathbf{V}_{l, j}\left(\mathbf{u}^{\prime}\right)\right|^{2} \\
& \leq \sum_{l=0}^{k} \sum_{j=0}^{N+1} C_{N}^{2} \sum_{n=0}^{k}\left(\left|\mathbf{u}_{n, j}-\mathbf{u}_{n, j}^{\prime}\right|^{2}+\left|\mathbf{u}_{n, j-1}-\mathbf{u}_{n, j-1}^{\prime}\right|^{2}\right) \\
& =2(k+1) C_{N}^{2}\left|\mathbf{u}-\mathbf{u}^{\prime}\right|^{2} .
\end{aligned}
$$

Step 2. Next we consider the local Lipschitz continuity of $\mathbf{W}$ with respect to the variable $\mathbf{u}$ for fixed $N \in \mathbb{N}$. Note that for any $l=0,1, \ldots, k, j=1,2, \ldots, N, \mathbf{u}, \mathbf{u}^{\prime} \in \mathbb{R}^{(k+1) \times(N+2)}$ with $|\mathbf{u}| \vee\left|\mathbf{u}^{\prime}\right| \leq M$,

$$
\mathbf{W}_{l, j}(\omega, t, \mathbf{u})-\mathbf{W}_{l, j}\left(\omega, t, \mathbf{u}^{\prime}\right)=E_{4}^{l, j}+E_{5}^{l, j}
$$

where

$$
\begin{aligned}
E_{4}^{l, j}:= & \int_{I_{j}} a\left(\omega, x, t, \sum_{n=0}^{k} \mathbf{u}_{n, j} \varphi_{n}^{j}(x), \sum_{n=0}^{k} \mathbf{V}_{n, j}(\mathbf{u}) \varphi_{n}^{j}(x)\right) \\
& \times \sum_{n=0}^{k}\left(\mathbf{V}_{n, j}(\mathbf{u})-\mathbf{V}_{n, j}\left(\mathbf{u}^{\prime}\right)\right) \varphi_{n}^{j}(x) \sum_{m=0}^{k} A_{l m}^{j,-1} \varphi_{m}^{j}(x) \mathrm{d} x, \\
E_{5}^{l, j}:= & \int_{I_{j}}\left[a\left(\omega, x, t, \sum_{n=0}^{k} \mathbf{u}_{n, j} \varphi_{n}^{j}(x), \sum_{n=0}^{k} \mathbf{V}_{n, j}(\mathbf{u}) \varphi_{n}^{j}(x)\right)\right. \\
& \left.-a\left(\omega, x, t, \sum_{n=0}^{k} \mathbf{u}_{n, j}^{\prime} \varphi_{n}^{j}(x), \sum_{n=0}^{k} \mathbf{V}_{n, j}\left(\mathbf{u}^{\prime}\right) \varphi_{n}^{j}(x)\right)\right] \\
& \times \sum_{n=0}^{k} \mathbf{V}_{n, j}\left(\mathbf{u}^{\prime}\right) \varphi_{n}^{j}(x) \sum_{m=0}^{k} A_{l m}^{j,-1} \varphi_{m}^{j}(x) \mathrm{d} x .
\end{aligned}
$$


From (H2) and (3.7), we have

$$
\begin{aligned}
\left|E_{4}^{l, j}\right| & \leq \Lambda \sum_{n=0}^{k} \int_{I_{j}}\left|\varphi_{n}^{j}(x)\right| \sum_{m=0}^{k}\left|\varphi_{m}^{j}(x)\right| \mathrm{d} x\left\|A^{j,-1}\right\|_{\infty}\left|\mathbf{V}_{n, j}(\mathbf{u})-\mathbf{V}_{n, j}\left(\mathbf{u}^{\prime}\right)\right| \\
& \leq \sum_{n=0}^{k} C_{N}\left|\mathbf{V}_{n, j}(\mathbf{u})-\mathbf{V}_{n, j}\left(\mathbf{u}^{\prime}\right)\right| \leq C_{N}\left(\sum_{n=0}^{k}\left|\mathbf{V}_{n, j}(\mathbf{u})-\mathbf{V}_{n, j}\left(\mathbf{u}^{\prime}\right)\right|^{2}\right)^{\frac{1}{2}} \\
& \leq C_{N}\left[\sum_{n=0}^{k}\left(\left|\mathbf{u}_{n, j}-\mathbf{u}_{n, j}^{\prime}\right|^{2}+\left|\mathbf{u}_{n, j-1}-\mathbf{u}_{n, j-1}^{\prime}\right|^{2}\right)\right]^{\frac{1}{2}} .
\end{aligned}
$$

Using Cauchy-Schwartz inequality, we have

$$
\begin{aligned}
\left|E_{5}^{l, j}\right|^{2} \leq & \int_{I_{j}} \mid a\left(\omega, x, t, \sum_{n=0}^{k} \mathbf{u}_{n, j} \varphi_{n}^{j}(x), \sum_{n=0}^{k} \mathbf{V}_{n, j}(\mathbf{u}) \varphi_{n}^{j}(x)\right) \\
& -\left.a\left(\omega, x, t, \sum_{n=0}^{k} \mathbf{u}_{n, j}^{\prime} \varphi_{n}^{j}(x), \sum_{n=0}^{k} \mathbf{V}_{n, j}\left(\mathbf{u}^{\prime}\right) \varphi_{n}^{j}(x)\right)\right|^{2} \mathrm{~d} x \\
& \times \int_{I_{j}}\left|\sum_{n=0}^{k} \mathbf{V}_{n, j}\left(\mathbf{u}^{\prime}\right) \varphi_{n}^{j}(x) \sum_{m=0}^{k} A_{l m}^{j,-1} \varphi_{m}^{j}(x)\right|^{2} \mathrm{~d} x \\
\leq & C_{N}\left|\mathbf{V}\left(\mathbf{u}^{\prime}\right)\right|^{2} \int_{I_{j}} L_{a}(M)^{2}\left(\left|\sum_{n=0}^{k}\left(\mathbf{u}_{n, j}-\mathbf{u}_{n, j}^{\prime}\right) \varphi_{n}^{j}(x)\right|+\left|\sum_{n=0}^{k}\left(\mathbf{V}_{n, j}(\mathbf{u})-\mathbf{V}_{n, j}\left(\mathbf{u}^{\prime}\right)\right) \varphi_{n}^{j}(x)\right|\right)^{2} \mathrm{~d} x \\
\leq & C_{N}(M) \sum_{n=0}^{k}\left(\left|\mathbf{u}_{n, j}-\mathbf{u}_{n, j}^{\prime}\right|^{2}+\left|\mathbf{V}_{n, j}(\mathbf{u})-\mathbf{V}_{n, j}\left(\mathbf{u}^{\prime}\right)\right|^{2}\right) \\
\leq & C_{N}(M) \sum_{n=0}^{k}\left(\left|\mathbf{u}_{n, j}-\mathbf{u}_{n, j}^{\prime}\right|^{2}+\left|\mathbf{u}_{n, j-1}-\mathbf{u}_{n, j-1}^{\prime}\right|^{2}\right)
\end{aligned}
$$

where $L_{a}(M)$ is the local Lipschitz constant of the function $a$.

For any $l=0,1, \ldots, k$ with $j=1,2, \ldots, N$, we have

$$
\begin{aligned}
\left|\mathbf{W}_{l, j}(\omega, t, \mathbf{u})-\mathbf{W}_{l, j}\left(\omega, t, \mathbf{u}^{\prime}\right)\right|^{2} & \leq 2\left(\left|E_{4}^{l, j}\right|^{2}+\left|E_{5}^{l, j}\right|^{2}\right) \\
& \leq C_{N}(M) \sum_{n=0}^{k}\left(\left|\mathbf{u}_{n, j}-\mathbf{u}_{n, j}^{\prime}\right|^{2}+\left|\mathbf{u}_{n, j-1}-\mathbf{u}_{n, j-1}^{\prime}\right|^{2}\right)
\end{aligned}
$$

Thus

$$
\left|\mathbf{W}(\omega, t, \mathbf{u})-\mathbf{W}\left(\omega, t, \mathbf{u}^{\prime}\right)\right|^{2} \leq C_{N}(M)\left|\mathbf{u}-\mathbf{u}^{\prime}\right|^{2}
$$

Step 3. We are now ready to prove the local Lipschitz continuity of the function $F$ for fixed $N \in \mathbb{N}$. Note that for any $l=0,1, \ldots, k$ and $j=1,2, \ldots, N$,

$$
F_{l, j}(\omega, t, \mathbf{u})-F_{l, j}\left(\omega, t, \mathbf{u}^{\prime}\right)=E_{6}^{l, j}+E_{7}^{l, j}+E_{8}^{l, j}+E_{9}^{l, j},
$$


where

$$
\begin{aligned}
E_{6}^{l, j}:= & -\int_{I_{j}} \sum_{n=0}^{k}\left(\mathbf{W}_{n, j}(\omega, t, \mathbf{u})-\mathbf{W}_{n, j}\left(\omega, t, \mathbf{u}^{\prime}\right)\right) \varphi_{n}^{j}(x) \sum_{m=0}^{k} A_{l m}^{j,-1} \varphi_{m x}^{j}(x) \mathrm{d} x, \\
E_{7}^{l, j}:= & \sum_{n=0}^{k}\left(\mathbf{W}_{n, j+1}(\omega, t, \mathbf{u})-\mathbf{W}_{n, j+1}\left(\omega, t, \mathbf{u}^{\prime}\right)\right) \varphi_{n}^{j+1}\left(x_{j+\frac{1}{2}}\right) \sum_{m=0}^{k} A_{l m}^{j,-1} \varphi_{m}^{j}\left(x_{j+\frac{1}{2}}\right), \\
E_{8}^{l, j}:= & -\sum_{n=0}^{k}\left(\mathbf{W}_{n, j}(\omega, t, \mathbf{u})-\mathbf{W}_{n, j}\left(\omega, t, \mathbf{u}^{\prime}\right)\right) \varphi_{n}^{j}\left(x_{j-\frac{1}{2}}\right) \sum_{m=0}^{k} A_{l m}^{j,-1} \varphi_{m}^{j}\left(x_{j-\frac{1}{2}}\right), \\
E_{9}^{l, j}:= & \int_{I_{j}}\left[\psi\left(\omega, x, t, \sum_{n=0}^{k} \mathbf{u}_{n, j} \varphi_{n}^{j}(x), \sum_{n=0}^{k} \mathbf{V}_{n, j}(\mathbf{u}) \varphi_{n}^{j}(x)\right)\right. \\
& \left.-\psi\left(\omega, x, t, \sum_{n=0}^{k} \mathbf{u}_{n, j}^{\prime} \varphi_{n}^{j}(x), \sum_{n=0}^{k} \mathbf{V}_{n, j}\left(\mathbf{u}^{\prime}\right) \varphi_{n}^{j}(x)\right)\right] \sum_{m=0}^{k} A_{l m}^{j,-1} \varphi_{m}^{j}(x) \mathrm{d} x .
\end{aligned}
$$

Similar to (3.7), we have

$$
\begin{aligned}
& \left|E_{6}^{l, j}\right|^{2}+\left|E_{7}^{l, j}\right|^{2}+\left|E_{8}^{l, j}\right|^{2} \\
\leq & C_{N}(M) \sum_{n=0}^{k}\left(\left|\mathbf{W}_{n, j}(\omega, t, \mathbf{u})-\mathbf{W}_{n, j}\left(\omega, t, \mathbf{u}^{\prime}\right)\right|^{2}+\left|\mathbf{W}_{n, j+1}(\omega, t, \mathbf{u})-\mathbf{W}_{n, j+1}\left(\omega, t, \mathbf{u}^{\prime}\right)\right|^{2}\right)
\end{aligned}
$$

In view of the Lipschitz continuity of $\mathbf{W}$ (see (3.11)), we have

$$
\begin{aligned}
\left|E_{6}^{l, j}\right|^{2}+\left|E_{7}^{l, j}\right|^{2}+\left|E_{8}^{l, j}\right|^{2} \leq & C_{N}(M) \sum_{n=0}^{k}\left(\left|\mathbf{u}_{n, j-1}-\mathbf{u}_{n, j-1}^{\prime}\right|^{2}\right. \\
& \left.+\left|\mathbf{u}_{n, j}-\mathbf{u}_{n, j}^{\prime}\right|^{2}+\left|\mathbf{u}_{n, j+1}-\mathbf{u}_{n, j+1}^{\prime}\right|^{2}\right) .
\end{aligned}
$$

Similar to Step 2, using the Lipschitz continuity of $\psi$ and $\mathbf{V}$, we have

$$
\begin{aligned}
\left|E_{9}^{l, j}\right|^{2} & \leq C_{N} \sum_{n=0}^{k}\left(\left|\mathbf{u}_{n, j}-\mathbf{u}_{n, j}^{\prime}\right|^{2}+\left|\mathbf{V}_{n, j}(\mathbf{u})-\mathbf{V}_{n, j}\left(\mathbf{u}^{\prime}\right)\right|^{2}\right) \\
& \leq C_{N} \sum_{n=0}^{k}\left(\left|\mathbf{u}_{n, j}-\mathbf{u}_{n, j}^{\prime}\right|^{2}+\left|\mathbf{u}_{n, j-1}-\mathbf{u}_{n, j-1}^{\prime}\right|^{2}\right) .
\end{aligned}
$$

At last, by the periodicity of the numerical solution $u_{h}$, we see that for any $N, M \in \mathbb{N}_{+}$, there exists a constant $L_{N}(M)$ such that, for all $(\omega, t) \in \Omega \times[0, T]$ and all $\mathbf{u}, \mathbf{u}^{\prime} \in \mathbb{R}^{(k+1) \times(N+2)}$ with $|\mathbf{u}| \vee\left|\mathbf{u}^{\prime}\right| \leq M$,

$$
\left|F(\omega, t, \mathbf{u})-F\left(\omega, t, \mathbf{u}^{\prime}\right)\right| \leq L_{N}(M)\left|\mathbf{u}-\mathbf{u}^{\prime}\right| .
$$

The proof is complete.

Similar to the proof of Lemma 3.2, we obtain that the coefficients of SDE (3.6) satisfy the linearly growing condition. 
Lemma 3.3. Let assumptions (H2)-(H4) hold. Then for any $N \in \mathbb{N}_{+}, F$ and $G$ are linearly growing in the variable $\mathbf{u}$, i.e., there exists a positive constant $C_{N}$ such that, for all $(\omega, t) \in \Omega \times[0, T]$ and all $\mathbf{u} \in \mathbb{R}^{(k+1) \times(N+2)}$,

$$
|F(\omega, t, \mathbf{u})| \vee|G(\omega, t, \mathbf{u})| \leq C_{N}(1+|\mathbf{u}|)
$$

where the constant $C_{N}$ may depend on $N$.

Proof. We only show the linear growth of $F$ for fixed $N \in \mathbb{N}$, and that of $G$ can be proved in a similar way.

Note that $\mathbf{V}(\mathbf{0})=\mathbf{0}$. Then by (3.8), we know that there exists a constant $C_{N}$ such that for any $\mathbf{u} \in$ $\mathbb{R}^{(k+1) \times(N+2),}$

$$
|\mathbf{V}(\mathbf{u})| \leq C_{N}|\mathbf{u}|
$$

By the fact $\mathbf{W}(\omega, t, \mathbf{0})=\mathbf{0}$, taking $\mathbf{u}^{\prime}=\mathbf{0}$ in (3.9) and (3.10), we have for any $l=0,1, \ldots, k, j=1,2, \ldots, N$

$$
\left|\mathbf{W}_{l, j}(\omega, t, \mathbf{u})\right|^{2} \leq C_{N} \sum_{n=0}^{k}\left(\left|\mathbf{u}_{n, j}\right|^{2}+\left|\mathbf{u}_{n, j-1}\right|^{2}\right)
$$

Thus

$$
|\mathbf{W}(\omega, t, \mathbf{u})|^{2}=\sum_{l=0}^{k} \sum_{j=0}^{N+1}\left|\mathbf{W}_{l, j}(\omega, t, \mathbf{u})\right|^{2} \leq C_{N}|\mathbf{u}|^{2} .
$$

Similar to the calculation in Step 3 of the proof of Lemma 3.2, by the linear growth of $\psi$, we have

$$
\begin{aligned}
\left|F_{l, j}(\omega, t, \mathbf{u})\right|^{2} & \leq C_{N} \sum_{n=0}^{k}\left(1+\left|\mathbf{u}_{n, j}\right|^{2}+\left|\mathbf{V}_{n, j}(\mathbf{u})\right|^{2}+\left|\mathbf{W}_{n, j}(\omega, t, \mathbf{u})\right|^{2}+\left|\mathbf{W}_{n, j+1}(\omega, t, \mathbf{u})\right|^{2}\right) \\
& \leq C_{N} \sum_{n=0}^{k}\left(1+\left|\mathbf{u}_{n, j-1}\right|^{2}+\left|\mathbf{u}_{n, j}\right|^{2}+\left|\mathbf{u}_{n, j+1}\right|^{2}\right)
\end{aligned}
$$

for any $l=0,1, \ldots, k$ and $j=1,2, \ldots, N$. Therefore,

$$
|F(\omega, t, \mathbf{u})| \leq C_{N}(1+|\mathbf{u}|) .
$$

By (3.2d), the initial condition of the SDE (3.6) is determined by $u_{0}$ as follows:

$$
\mathbf{u}_{l, j}(\omega, 0):=\sum_{m=0}^{k} A_{l m}^{j,-1} \int_{I_{j}} u_{0}(x) \varphi_{m}^{j}(x) \mathrm{d} x .
$$

Since $u_{0}$ is deterministic, we know that $\mathbf{u}(\cdot, 0)$ is a deterministic matrix. Thus for any $p \geq 1$, we have

$$
\mathbb{E}\left[|\mathbf{u}(\cdot, 0)|^{p}\right]<\infty .
$$

The following lemma is a classical result of stochastic differential equations. See e.g. [32], Chapter 3.

Lemma 3.4. If the initial value $\mathbf{u}(\cdot, 0)$ is $L^{p}(\Omega)$-integrable, and the coefficients $F, G$ are locally Lipschitz continuous and linearly growing, then the underlying $S D E$ admits a unique solution $\{\mathbf{u}(t)\}_{0 \leq t \leq T}$ such that for any $p \geq 1$,

$$
\mathbb{E}\left[\sup _{0 \leq t \leq T}|\mathbf{u}(t)|^{p}\right]<\infty
$$


Thus, by virtue of (3.16), Lemmas 3.2 and 3.3, we know that for any fixed $N \in \mathbb{N}_{+}$, SDE (3.6) has a unique solution $\{\mathbf{u}(t)\}_{0 \leq t \leq T}$ such that (3.17) holds. By the linear growth of the functions $\mathbf{V}$ and $\mathbf{W}$ (see (3.13) and (3.14)), we get that for any $p \geq 1$,

$$
\mathbb{E}\left[\sup _{0 \leq t \leq T}|\mathbf{v}(t)|^{p}\right]<\infty, \quad \mathbb{E}\left[\sup _{0 \leq t \leq T}|\mathbf{w}(t)|^{p}\right]<\infty .
$$

\section{Stability ANALYSis FOR THE FUlly NONLINEAR EQUATIONS}

We have known that the approximating equation (3.2) has a unique solution $\left(u_{h}, v_{h}, w_{h}\right)$ for any fixed $N \in \mathbb{N}_{+}$, where $\left(u_{h}, v_{h}, w_{h}\right)^{T}(\omega, \cdot, t) \in\left(V_{h}\right)^{3}$ for each $(\omega, t) \in \Omega \times[0, T]$. Next we show the stability result for the numerical solutions. We first consider the nondegenerate case that $2 \alpha>C_{4}^{2}$.

Theorem 4.1. Suppose that the assumptions (H1)-(H4) are satisfied. Moreover, we assume that $2 \alpha>C_{4}^{2}$. Then there exists a constant $C>0$ such that

$$
\sup _{0 \leq t \leq T} \mathbb{E}\left[\left\|u_{h}(\cdot, t)\right\|^{2}\right]+\mathbb{E}\left[\int_{0}^{T}\left\|v_{h}(\cdot, s)\right\|^{2} \mathrm{~d} s\right] \leq C\left(1+\left\|u_{h}(\cdot, 0)\right\|^{2}\right),
$$

where the constant $C$ is independent of $h$, and depends on the terminal time $T$.

Proof. For any $N \in \mathbb{N}_{+}$and $(\omega, t) \in \Omega \times[0, T]$, by setting $r_{h}=u_{h}(\omega, \cdot, t)$ in $(3.2 \mathrm{a}), z_{h}=w_{h}(\omega, \cdot, t)$ in $(3.2 \mathrm{~b})$, and multiplying $(3.2 \mathrm{~b})$ with $\mathrm{d} t$, adding the resulting equations, we have

$$
\begin{aligned}
\int_{I_{j}} u_{h}(x, t) \mathrm{d} u_{h}(x, t) \mathrm{d} x+ & \int_{I_{j}} v_{h}(x, t) w_{h}(x, t) \mathrm{d} x \mathrm{~d} t \\
= & \left\{\int_{I_{j}} \psi\left(x, t, u_{h}(x, t), v_{h}(x, t)\right) u_{h}(x, t) \mathrm{d} x\right. \\
& -\int_{I_{j}} w_{h}(x, t) u_{h x}(x, t) \mathrm{d} x+w_{h, j+\frac{1}{2}}^{+} u_{h, j+\frac{1}{2}}^{-}-w_{h, j-\frac{1}{2}}^{+} u_{h, j-\frac{1}{2}}^{+} \\
& \left.-\int_{I_{j}} u_{h}(x, t) w_{h x}(x, t) \mathrm{d} x+u_{h, j+\frac{1}{2}}^{-} w_{h, j+\frac{1}{2}}^{-}-u_{h, j-\frac{1}{2}}^{-} w_{h, j-\frac{1}{2}}^{+}\right\} \mathrm{d} t \\
& +\int_{I_{j}} g\left(x, t, u_{h}(x, t), v_{h}(x, t)\right) u_{h}(x, t) \mathrm{d} x \mathrm{~d} W_{t},
\end{aligned}
$$

where

$$
u_{h, j+\frac{1}{2}}^{ \pm}=u_{h}\left(\omega, x_{j+\frac{1}{2}}^{ \pm}, t\right), \quad w_{h, j+\frac{1}{2}}^{ \pm}=w_{h}\left(\omega, x_{j+\frac{1}{2}}^{ \pm}, t\right)
$$

For simplicity of notation, for $j=1,2, \ldots, N$ and piece-wisely smooth functions $u$ and $v$, we define

$$
H_{j}^{ \pm}(u, v):=-\int_{I_{j}} u(x) v_{x}(x) \mathrm{d} x+u\left(x_{j+\frac{1}{2}}^{ \pm}\right) v\left(x_{j+\frac{1}{2}}^{-}\right)-u\left(x_{j-\frac{1}{2}}^{ \pm}\right) v\left(x_{j-\frac{1}{2}}^{+}\right) .
$$

Thus we have

$$
\begin{aligned}
\int_{I_{j}} u_{h}(x, t) \mathrm{d} u_{h}(x, t) \mathrm{d} x+ & \int_{I_{j}} v_{h}(x, t) w_{h}(x, t) \mathrm{d} x \mathrm{~d} t \\
= & \left\{\int_{I_{j}} \psi\left(x, t, u_{h}(x, t), v_{h}(x, t)\right) u_{h}(x, t) \mathrm{d} x\right. \\
& \left.+H_{j}^{+}\left(w_{h}(\omega, \cdot, t), u_{h}(\omega, \cdot, t)\right)+H_{j}^{-}\left(u_{h}(\omega, \cdot, t), w_{h}(\omega, \cdot, t)\right)\right\} \mathrm{d} t \\
& +\int_{I_{j}} g\left(x, t, u_{h}(x, t), v_{h}(x, t)\right) u_{h}(x, t) \mathrm{d} x \mathrm{~d} W_{t} .
\end{aligned}
$$


By taking $p_{h}=v_{h}(\omega, \cdot, t)$ in $(3.2 \mathrm{c})$, it holds that

$$
\mathbb{E}\left[\int_{0}^{t} \int_{0}^{2 \pi} v_{h}(x, s) w_{h}(x, s) \mathrm{d} x \mathrm{~d} s\right]=\mathbb{E}\left[\int_{0}^{t} \int_{0}^{2 \pi} a\left(x, s, u_{h}(x, s), v_{h}(x, s)\right)\left|v_{h}(x, s)\right|^{2} \mathrm{~d} x \mathrm{~d} s\right] .
$$

Using Itô's formula, we have

$$
\left|u_{h}(x, t)\right|^{2}=\left|u_{h}(x, 0)\right|^{2}+2 \int_{0}^{t} u_{h}(x, s) \mathrm{d} u_{h}(x, s)+\left\langle u_{h}(x, \cdot), u_{h}(x, \cdot)\right\rangle_{t} .
$$

Thus, after summing over $j$ from 1 to $N$ in (4.2), integrating in time from 0 to $t$ and taking expectation, we have

$$
\begin{aligned}
\mathbb{E}\left[\left\|u_{h}(\cdot, t)\right\|^{2}\right] & +2 \mathbb{E}\left[\int_{0}^{t} \int_{0}^{2 \pi} a\left(x, s, u_{h}(x, s), v_{h}(x, s)\right)\left|v_{h}(x, s)\right|^{2} \mathrm{~d} x \mathrm{~d} s\right] \\
& =\left\|u_{h}(\cdot, 0)\right\|^{2}+\mathcal{T}_{1}(t)+\mathcal{T}_{2}(t)+\mathcal{T}_{3}(t)+\mathcal{T}_{4}(t),
\end{aligned}
$$

where

$$
\begin{aligned}
& \mathcal{T}_{1}(t)=\mathbb{E}\left[\int_{0}^{2 \pi}\left\langle u_{h}(x, \cdot), u_{h}(x, \cdot)\right\rangle_{t} \mathrm{~d} x\right] \\
& \mathcal{T}_{2}(t)=2 \mathbb{E}\left[\int_{0}^{t} \int_{0}^{2 \pi} g\left(x, s, u_{h}(x, s), v_{h}(x, s)\right) u_{h}(x, s) \mathrm{d} x \mathrm{~d} W_{s}\right], \\
& \mathcal{T}_{3}(t)=2 \mathbb{E}\left[\int_{0}^{t} \int_{0}^{2 \pi} \psi\left(x, s, u_{h}(x, s), v_{h}(x, s)\right) u_{h}(x, s) \mathrm{d} x \mathrm{~d} s\right],
\end{aligned}
$$

and

$$
\mathcal{T}_{4}(t)=2 \mathbb{E}\left[\int_{0}^{t} \sum_{j=1}^{N}\left\{H_{j}^{+}\left(w_{h}(\omega, \cdot, s), u_{h}(\omega, \cdot, s)\right)+H_{j}^{-}\left(u_{h}(\omega, \cdot, s), w_{h}(\omega, \cdot, s)\right)\right\} \mathrm{d} s\right] .
$$

The terms $\mathcal{T}_{i}(t)$ for $i=1, \ldots, 4$ are estimated as follows.

- The estimate of $\mathcal{T}_{1}(t)$.

In view of $(3.2 \mathrm{a})$ and $(3.2 \mathrm{~d})$, we have for any $r_{h} \in V_{h}$,

$$
\int_{I_{j}} r_{h}(x) u_{h}(x, t) \mathrm{d} x=\Xi(\omega, t)+\int_{0}^{t} \int_{I_{j}} g\left(x, s, u_{h}(x, s), v_{h}(x, s)\right) r_{h}(x) \mathrm{d} x \mathrm{~d} W_{s} .
$$

where

$$
\begin{aligned}
\Xi(\omega, t):= & \int_{0}^{t}\left\{-\int_{I_{j}} w_{h}(\omega, x, s) r_{h x}(x) \mathrm{d} x+\widehat{w}_{j+\frac{1}{2}}(\omega, s) r_{h}\left(x_{j+\frac{1}{2}}^{-}\right)-\widehat{w}_{j-\frac{1}{2}}(\omega, s) r_{h}\left(x_{j-\frac{1}{2}}^{+}\right)\right. \\
& \left.+\int_{I_{j}} \psi\left(\omega, x, s, u_{h}(\omega, x, s), v_{h}(\omega, x, s)\right) r_{h}(x) \mathrm{d} x\right\} \mathrm{d} s+\int_{I_{j}} r_{h}(x) u_{0}(x) \mathrm{d} x .
\end{aligned}
$$

Note that $\Xi$ is a continuous stochastic process with bounded total variation. By (2.5), for any continuous semimartingale $Y$, we have

$$
\langle\Xi, Y\rangle_{t} \equiv 0
$$


which leads to for any $r_{h} \in V_{h}$,

$$
\begin{aligned}
\int_{I_{j}} r_{h}(x)\left\langle u_{h}(x, \cdot), Y\right\rangle_{t} \mathrm{~d} x & =\left\langle\int_{I_{j}} r_{h}(x) u_{h}(x, \cdot) \mathrm{d} x, Y\right\rangle_{t} \\
& =\left\langle\int_{0} \int_{I_{j}} g\left(x, s, u_{h}(x, s), v_{h}(x, s)\right) r_{h}(x) \mathrm{d} x \mathrm{~d} W_{s}, Y\right\rangle_{t} .
\end{aligned}
$$

It turns out that

$$
\begin{aligned}
\int_{I_{j}}\left\langle u_{h}(x, \cdot), u_{h}(x, \cdot)\right\rangle_{t} \mathrm{~d} x & =\int_{I_{j}}\left\langle u_{h}(x, \cdot), \sum_{l=0}^{k} \mathbf{u}_{l, j}(\cdot) \varphi_{l}^{j}(x)\right\rangle_{t} \mathrm{~d} x \\
& =\sum_{l=0}^{k} \int_{I_{j}} \varphi_{l}^{j}(x)\left\langle u_{h}(x, \cdot), \mathbf{u}_{l, j}(\cdot)\right\rangle_{t} \mathrm{~d} x \\
& =\sum_{l=0}^{k}\left\langle\int_{0} \int_{I_{j}} g\left(x, s, u_{h}(x, s), v_{h}(x, s)\right) \varphi_{l}^{j}(x) \mathrm{d} x \mathrm{~d} W_{s}, \mathbf{u}_{l, j}(\cdot)\right\rangle_{t},
\end{aligned}
$$

where $\left\{\varphi_{l}^{j}, l=0,1, \ldots, k\right\}$ is a basis of $P^{k}\left(I_{j}\right)$.

According to (2.4) and the properties of the $L^{2}$ projection, we have

$$
\begin{aligned}
\int_{I_{j}}\left\langle u_{h}(x, \cdot), u_{h}(x, \cdot)\right\rangle_{t} \mathrm{~d} x & =\sum_{l=0}^{k} \int_{0}^{t} \int_{I_{j}} g\left(x, s, u_{h}(x, s), v_{h}(x, s)\right) \varphi_{l}^{j}(x) \mathrm{d} x \mathrm{~d}\left\langle W, \mathbf{u}_{l, j}(\cdot)\right\rangle_{s} \\
& =\sum_{l=0}^{k} \int_{0}^{t} \int_{I_{j}} \mathcal{P}\left[g\left(\cdot, s, u_{h}(\cdot, s), v_{h}(\cdot, s)\right)\right](x) \varphi_{l}^{j}(x) \mathrm{d} x \mathrm{~d}\left\langle W, \mathbf{u}_{l, j}(\cdot)\right\rangle_{s} \\
& =\int_{I_{j}} \int_{0}^{t} \sum_{l=0}^{k} \mathcal{P}\left[g\left(\cdot, s, u_{h}(\cdot, s), v_{h}(\cdot, s)\right)\right](x) \varphi_{l}^{j}(x) \mathrm{d}\left\langle W, \mathbf{u}_{l, j}(\cdot)\right\rangle_{s} \mathrm{~d} x \\
& =\int_{I_{j}} \int_{0}^{t} \mathcal{P}\left[g\left(\cdot, s, u_{h}(\cdot, s), v_{h}(\cdot, s)\right)\right](x) \mathrm{d}\left\langle W, \sum_{l=0}^{k} \mathbf{u}_{l, j}(\cdot) \varphi_{l}^{j}(x)\right\rangle_{s} \mathrm{~d} x \\
& =\int_{I_{j}}\left\langle\int_{0}^{\cdot} \mathcal{P}\left[g\left(\cdot, s, u_{h}(\cdot, s), v_{h}(\cdot, s)\right)\right](x) \mathrm{d} W_{s}, u_{h}(x, \cdot)\right\rangle_{t} \mathrm{~d} x .
\end{aligned}
$$

Since $\mathcal{P}\left[g\left(\cdot, s, u_{h}(\cdot, s), v_{h}(\cdot, s)\right)\right] \in V_{h}$ for any $(\omega, s) \in \Omega \times[0, T]$, we have

$$
\mathcal{P}\left[g\left(\omega, \cdot, s, u_{h}(\omega, \cdot, s), v_{h}(\omega, \cdot, s)\right)\right](x)=\sum_{l=0}^{k} \mathbf{g}_{l, j}(\omega, s) \varphi_{l}^{j}(x), \quad x \in I_{j} .
$$

By (4.3), we get

$$
\begin{aligned}
\int_{I_{j}}\left\langle u_{h}(x, \cdot), u_{h}(x, \cdot)\right\rangle_{t} \mathrm{~d} x & =\int_{I_{j}}\left\langle\int_{0} \sum_{l=0}^{k} \mathbf{g}_{l, j}(s) \varphi_{l}^{j}(x) \mathrm{d} W_{s}, u_{h}(x, \cdot)\right\rangle_{t} \mathrm{~d} x \\
& =\sum_{l=0}^{k} \int_{I_{j}} \varphi_{l}^{j}(x)\left\langle u_{h}(x, \cdot), \int_{0} \mathbf{g}_{l, j}(s) \mathrm{d} W_{s}\right\rangle_{t} \mathrm{~d} x
\end{aligned}
$$




$$
\begin{aligned}
& =\sum_{l=0}^{k}\left\langle\int_{0} \int_{I_{j}} g\left(x, s, u_{h}(x, s), v_{h}(x, s)\right) \varphi_{l}^{j}(x) \mathrm{d} x \mathrm{~d} W_{s}, \int_{0} \mathbf{g}_{l, j}(s) \mathrm{d} W_{s}\right\rangle_{t} \\
& =\sum_{l=0}^{k} \int_{0}^{t} \int_{I_{j}} g\left(x, s, u_{h}(x, s), v_{h}(x, s)\right) \varphi_{l}^{j}(x) \mathrm{d} x \mathbf{g}_{l, j}(s) d\langle W, W\rangle_{s} \\
& =\int_{0}^{t} \int_{I_{j}} g\left(x, s, u_{h}(x, s), v_{h}(x, s)\right) \sum_{l=0}^{k} \mathbf{g}_{l, j}(s) \varphi_{l}^{j}(x) \mathrm{d} x \mathrm{~d} s \\
& =\int_{0}^{t} \int_{I_{j}} g\left(x, s, u_{h}(x, s), v_{h}(x, s)\right) \mathcal{P}\left[g\left(\cdot, s, u_{h}(\cdot, s), v_{h}(\cdot, s)\right)\right](x) \mathrm{d} x \mathrm{~d} s .
\end{aligned}
$$

After summing over $j$ from 1 to $N$, by the Cauchy-Schwartz inequality we have

$$
\int_{0}^{2 \pi}\left\langle u_{h}(x, \cdot), u_{h}(x, \cdot)\right\rangle_{t} \mathrm{~d} x \leq \int_{0}^{t} \int_{0}^{2 \pi}\left|g\left(x, s, u_{h}(x, s), v_{h}(x, s)\right)\right|^{2} \mathrm{~d} x \mathrm{~d} s .
$$

According to (H4), after taking expectation, we have

$$
\begin{aligned}
\mathcal{T}_{1}(t) & =\mathbb{E}\left[\int_{0}^{2 \pi}\left\langle u_{h}(x, \cdot), u_{h}(x, \cdot)\right\rangle_{t} \mathrm{~d} x\right] \\
& \leq \mathbb{E}\left[\int_{0}^{t} \int_{0}^{2 \pi}\left|g\left(x, s, u_{h}(x, s), v_{h}(x, s)\right)\right|^{2} \mathrm{~d} x \mathrm{~d} s\right] \\
& \leq C_{3}^{2} \mathbb{E}\left[\int_{0}^{t} \int_{0}^{2 \pi}\left(1+\left|u_{h}(x, s)\right|^{2}\right) \mathrm{d} x \mathrm{~d} s\right]+C_{4}^{2} \mathbb{E}\left[\int_{0}^{t} \int_{0}^{2 \pi}\left|v_{h}(x, s)\right|^{2} \mathrm{~d} x \mathrm{~d} s\right] \\
& =2 \pi T C_{3}^{2}+C_{3}^{2} \int_{0}^{t} \mathbb{E}\left[\left\|u_{h}(\cdot, s)\right\|^{2}\right] \mathrm{d} s+C_{4}^{2} \int_{0}^{t} \mathbb{E}\left[\left\|v_{h}(\cdot, s)\right\|^{2}\right] \mathrm{d} s .
\end{aligned}
$$

- The estimate of $\mathcal{T}_{2}(t)$.

From (3.17) and (3.18), we have for any fixed $N \in \mathbb{N}_{+}$,

$$
\mathbb{E}\left[\sup _{0 \leq s \leq T}\left\{\left\|u_{h}(\cdot, s)\right\|^{2}+\left\|v_{h}(\cdot, s)\right\|^{2}\right\}\right]<\infty .
$$

Thus by (H4) and the Cauchy-Schwartz inequality we know that

$$
\begin{aligned}
& \mathbb{E}\left[\left(\int_{0}^{T}\left|\int_{0}^{2 \pi} g\left(x, s, u_{h}(x, s), v_{h}(x, s)\right) u_{h}(x, s) \mathrm{d} x\right|^{2} \mathrm{~d} s\right)^{\frac{1}{2}}\right] \\
& \leq \mathbb{E}\left[\left(\int_{0}^{T}\left\|u_{h}(\cdot, s)\right\|^{2} \int_{0}^{2 \pi}\left|g\left(x, s, u_{h}(x, s), v_{h}(x, s)\right)\right|^{2} \mathrm{~d} x \mathrm{~d} s\right)^{\frac{1}{2}}\right] \\
& \leq C \mathbb{E}\left[\sup _{0 \leq s \leq T}\left\|u_{h}(\cdot, s)\right\|\left(\int_{0}^{T} \int_{0}^{2 \pi}\left(1+\left|u_{h}(x, s)\right|^{2}+\left|v_{h}(x, s)\right|^{2}\right) \mathrm{d} x \mathrm{~d} s\right)^{\frac{1}{2}}\right] \\
& \leq C\left(\mathbb{E}\left[\sup _{0 \leq s \leq T}\left\|u_{h}(\cdot, s)\right\|^{2}\right]\right)^{\frac{1}{2}}\left(\mathbb{E}\left[\int_{0}^{T}\left(1+\left\|u_{h}(\cdot, s)\right\|^{2}+\left\|v_{h}(\cdot, s)\right\|^{2}\right) \mathrm{d} s\right]\right)^{\frac{1}{2}}<\infty .
\end{aligned}
$$


According to Lemma 2.1, the process

$$
\left\{\int_{0}^{t} \int_{0}^{2 \pi} g\left(x, s, u_{h}(x, s), v_{h}(x, s)\right) u_{h}(x, s) \mathrm{d} x \mathrm{~d} W_{s}, \quad 0 \leq t \leq T\right\}
$$

is a martingale. It turns out that

$$
\mathcal{T}_{2}(t)=2 \mathbb{E}\left[\int_{0}^{t} \int_{0}^{2 \pi} g\left(x, s, u_{h}(x, s), v_{h}(x, s)\right) u_{h}(x, s) \mathrm{d} x \mathrm{~d} W_{s}\right]=0 .
$$

- The estimate of $\mathcal{T}_{3}(t)$.

Note that according to (H3), for any $\varepsilon>0$, it holds that

$$
\begin{aligned}
\int_{0}^{2 \pi} & \psi\left(x, t, u_{h}(x, t), v_{h}(x, t)\right) u_{h}(x, t) \mathrm{d} x \\
& \leq \frac{\varepsilon}{2} \int_{0}^{2 \pi}\left|\psi\left(x, t, u_{h}(x, t), v_{h}(x, t)\right)\right|^{2} \mathrm{~d} x+\frac{1}{2 \varepsilon} \int_{0}^{2 \pi}\left|u_{h}(x, t)\right|^{2} \mathrm{~d} x \\
& \leq \frac{\varepsilon}{2} \int_{0}^{2 \pi}\left[B_{2}^{2}+B_{2}^{2}\left|u_{h}(x, t)\right|^{2}+B_{3}^{2}\left|v_{h}(x, t)\right|^{2}\right] \mathrm{d} x+\frac{1}{2 \varepsilon}\left\|u_{h}(\cdot, t)\right\|^{2} \\
& =\frac{\varepsilon B_{3}^{2}}{2}\left\|v_{h}(\cdot, t)\right\|^{2}+\varepsilon \pi B_{2}^{2}+\left(\frac{\varepsilon B_{2}^{2}}{2}+\frac{1}{2 \varepsilon}\right)\left\|u_{h}(\cdot, t)\right\|^{2} .
\end{aligned}
$$

Then

$$
\begin{aligned}
\mathcal{T}_{3}(t) & =2 \mathbb{E}\left[\int_{0}^{t} \int_{0}^{2 \pi} \psi\left(x, s, u_{h}(x, s), v_{h}(x, s)\right) u_{h}(x, s) \mathrm{d} x \mathrm{~d} s\right] \\
& \leq \varepsilon B_{3}^{2} \mathbb{E}\left[\int_{0}^{t}\left\|v_{h}(\cdot, s)\right\|^{2} \mathrm{~d} s\right]+2 \varepsilon \pi B_{2}^{2} T+\left(\varepsilon B_{2}^{2}+\frac{1}{\varepsilon}\right) \int_{0}^{t} \mathbb{E}\left[\left\|u_{h}(\cdot, s)\right\|^{2}\right] \mathrm{d} s .
\end{aligned}
$$

- The estimate of $\mathcal{T}_{4}(t)$.

According to the periodicity, we have for any $u, v \in V_{h}$,

$$
\begin{aligned}
\sum_{j=1}^{N} & {\left[H_{j}^{+}(u, v)+H_{j}^{-}(v, u)\right] } \\
& =\sum_{j=1}^{N}\left(-u_{j+\frac{1}{2}}^{-} v_{j+\frac{1}{2}}^{-}+u_{j-\frac{1}{2}}^{+} v_{j-\frac{1}{2}}^{+}+u_{j+\frac{1}{2}}^{+} v_{j+\frac{1}{2}}^{-}-u_{j-\frac{1}{2}}^{+} v_{j-\frac{1}{2}}^{+}+v_{j+\frac{1}{2}}^{-} u_{j+\frac{1}{2}}^{-}-v_{j-\frac{1}{2}}^{-} u_{j-\frac{1}{2}}^{+}\right) \\
& =\sum_{j=1}^{N}\left(-u^{-} v^{-}+u^{+} v^{+}+u^{+} v^{-}-u^{+} v^{+}+v^{-} u^{-}-v^{-} u^{+}\right)_{j+\frac{1}{2}}=0 .
\end{aligned}
$$

Thus

$$
\mathcal{T}_{4}(t)=2 \mathbb{E}\left[\int_{0}^{t} \sum_{j=1}^{N}\left\{H_{j}^{+}\left(w_{h}(\omega, \cdot, s), u_{h}(\omega, \cdot, s)\right)+H_{j}^{-}\left(u_{h}(\omega, \cdot, s), w_{h}(\omega, \cdot, s)\right)\right\} \mathrm{d} s\right]=0
$$


Concluding the above, we get that for any $\varepsilon>0$,

$$
\begin{aligned}
\mathbb{E}\left[\left\|u_{h}(\cdot, t)\right\|^{2}\right]+ & 2 \mathbb{E}\left[\int_{0}^{t} \int_{0}^{2 \pi} a\left(x, s, u_{h}(x, s), v_{h}(x, s)\right)\left|v_{h}(x, s)\right|^{2} \mathrm{~d} x \mathrm{~d} s\right] \\
\leq & \mathbb{E}\left[\left\|u_{h}(\cdot, 0)\right\|^{2}\right]+2 \pi T C_{3}^{2}+2 \varepsilon \pi B_{2}^{2} T+\left(C_{3}^{2}+\varepsilon B_{2}^{2}+\frac{1}{\varepsilon}\right) \int_{0}^{t} \mathbb{E}\left[\left\|u_{h}(\cdot, s)\right\|^{2}\right] \mathrm{d} s \\
& +\left(C_{4}^{2}+\varepsilon B_{3}^{2}\right) \mathbb{E}\left[\int_{0}^{t}\left\|v_{h}(\cdot, s)\right\|^{2} \mathrm{~d} s\right] .
\end{aligned}
$$

By (H2) we know that $a(\omega, x, s, u, v) \geq \alpha$. Since $2 \alpha>C_{4}^{2}$, we take

$$
\varepsilon:=\frac{1}{B_{3}^{2}}\left(\alpha-\frac{1}{2} C_{4}^{2}\right)>0 .
$$

Then there exists a positive constant $C$ which is independent of $h$, such that for any $t \in[0, T]$,

$$
\mathbb{E}\left[\left\|u_{h}(\cdot, t)\right\|^{2}\right]+\left(\alpha-\frac{1}{2} C_{4}^{2}\right) \mathbb{E}\left[\int_{0}^{t}\left\|v_{h}(\cdot, s)\right\|^{2} \mathrm{~d} s\right] \leq\left\|u_{h}(\cdot, 0)\right\|^{2}+C+C \int_{0}^{t} \mathbb{E}\left[\left\|u_{h}(\cdot, s)\right\|^{2}\right] \mathrm{d} s .
$$

Using Gronwall's inequality, we have for any $t \in[0, T]$,

$$
\mathbb{E}\left[\left\|u_{h}(\cdot, t)\right\|^{2}\right]+\mathbb{E}\left[\int_{0}^{t}\left\|v_{h}(\cdot, s)\right\|^{2} \mathrm{~d} s\right] \leq C\left(1+\left\|u_{h}(\cdot, 0)\right\|^{2}\right) e^{C t} .
$$

This completes the proof.

Our above stability result does not involve the regularity of the solution of the original SPDEs, and applies to the degenerate case of $2 \alpha=C_{4}^{2}$. In particular, it applies to the special case of $\alpha=0$, as long as the constants $C_{4}$ and $B_{3}$ vanish. When the function $\psi$ is uniformly bounded with respect to the last argument, as an immediate consequence of (4.6) with $\varepsilon=1$, we have the following stability result for the degenerate case.

Theorem 4.2. If the assumptions (H1)-(H4) hold with $2 \alpha=C_{4}^{2}$ and $B_{3}=0$, then there exists a constant $C>0$ such that

$$
\sup _{0 \leq t \leq T} \mathbb{E}\left[\left\|u_{h}(\cdot, t)\right\|^{2}\right] \leq C\left(1+\left\|u_{h}(\cdot, 0)\right\|^{2}\right),
$$

where the constant $C$ is independent of $h$ and depends on the terminal time $T$.

Remark 4.3. The inequality $2 \alpha \geq C_{4}^{2}$, used to derive our stability of the numerical schemes, is the so-called stochastic parabolicity, which is crucial in the theory of parabolic SPDEs (see e.g. [37,39]). The numerical experiments in Section 7 also illustrate its necessity.

Remark 4.4. Following the ideas in $[26,30]$, we can extend our method to the more general SPDEs with a nonlinear convection term $f(\cdot)$ :

$$
\left\{\begin{array}{lr}
\mathrm{d} u=\left\{\left[a\left(\cdot, x, t, u, u_{x}\right) u_{x}\right]_{x}-f(u)_{x}+\psi\left(\cdot, x, t, u, u_{x}\right)\right\} \mathrm{d} t+g\left(\cdot, x, t, u, u_{x}\right) \mathrm{d} W_{t}, \\
& (x, t) \in[0,2 \pi] \times(0, T] ; \\
u(x, 0)=u_{0}(x), & x \in[0,2 \pi],
\end{array}\right.
$$

where $f(\cdot)$ is locally Lipschitz continuous and can be super-linearly growing, such as $f(u)=\frac{u^{2}}{2}$ considered in Section 7.3. Using the monotone numerical flux for the nonlinear convection term $f(\cdot)$, we can also get similar stability results even for the degenerate case. A numerical test is reported for the stochastic viscous Burgers equation in Section 7.3. 


\section{Optimal ERror estimates For SEMilineAR EQUATIONS}

In this section, we consider the convergence of numerical methods for strong solutions with enough smoothness and integrability. We prove the optimal error estimates $\left(\mathcal{O}\left(h^{k+1}\right)\right)$ for the following semi-linear SPDE :

$$
\begin{cases}\mathrm{d} u=\left[a u_{x x}+\psi\left(\cdot, x, t, u, u_{x}\right)\right] \mathrm{d} t+g\left(\cdot, x, t, u, u_{x}\right) \mathrm{d} W_{t}, & (x, t) \in[0,2 \pi] \times(0, T] ; \\ u(x, 0)=u_{0}(x), & x \in[0,2 \pi]\end{cases}
$$

when the leading coefficient $a$ is a positive constant. We rewrite (5.1) as follows:

$$
\begin{cases}\mathrm{d} u=\left[a v_{x}+\psi(\cdot, x, t, u, v)\right] \mathrm{d} t+g(\cdot, x, t, u, v) \mathrm{d} W_{t}, & (x, t) \in[0,2 \pi] \times(0, T] \\ v(x, t)=u_{x}(x, t), & (x, t) \in[0,2 \pi] \times(0, T] \\ u(x, 0)=u_{0}(x), & x \in[0,2 \pi]\end{cases}
$$

From (3.2c), we have that $w_{h}=a v_{h}$. Then the LDG method (3.2) is written as follows. For any $(\omega, t) \in$ $\Omega \times[0, T]$, find $u_{h}(\omega, \cdot, t), v_{h}(\omega, \cdot, t) \in V_{h}$ such that for any $r_{h}, z_{h} \in V_{h}$,

$$
\begin{aligned}
\int_{I_{j}} r_{h}(x) \mathrm{d} u_{h}(\omega, x, t) \mathrm{d} x= & {\left[a H_{j}^{+}\left(v_{h}(\omega, \cdot, t), r_{h}\right)+\int_{I_{j}} \psi\left(\omega, x, t, u_{h}(\omega, x, t), v_{h}(\omega, x, t)\right) r_{h}(x) \mathrm{d} x\right] \mathrm{d} t } \\
& +\int_{I_{j}} g\left(\omega, x, t, u_{h}(\omega, x, t), v_{h}(\omega, x, t)\right) r_{h}(x) \mathrm{d} x \mathrm{~d} W_{t} \\
& \int_{I_{j}} v_{h}(\omega, x, t) z_{h}(x) \mathrm{d} x=H_{j}^{-}\left(u_{h}(\omega, \cdot, t), z_{h}\right)
\end{aligned}
$$

where the bilinear functionals $H_{j}^{ \pm}$are defined by (4.1). Then, we state the error estimates of the semi-discrete LDG scheme (5.3).

Theorem 5.1. Suppose that $u_{0} \in H^{k+1}$, assumptions (H3) and (H4) hold with $2 a>C_{2}^{2}$, and equation (5.1) has a unique strong solution $u$ such that

(H5) $u \in L^{2}\left(\Omega \times[0, T] ; H^{k+3}\right) \bigcap \mathcal{S}^{2}\left(\Omega \times[0, T] ; L^{2}\right) \bigcap L^{\infty}\left(0, T ; L^{2}\left(\Omega ; H^{k+1}\right)\right) ;$

(H6) $\psi\left(\cdot, u(\cdot), u_{x}(\cdot)\right), g\left(\cdot, u(\cdot), u_{x}(\cdot)\right) \in L^{2}\left(\Omega \times[0, T] ; H^{k+1}\right)$.

Then, there is a positive constant $C$ such that

$$
\sup _{t \in[0, T]}\left(\mathbb{E}\left[\left\|u(\cdot, t)-u_{h}(\cdot, t)\right\|^{2}\right]\right)^{\frac{1}{2}}+\left(\mathbb{E}\left[\int_{0}^{T}\left\|u_{x}(\cdot, s)-v_{h}(\cdot, s)\right\|^{2} \mathrm{~d} s\right]\right)^{\frac{1}{2}} \leq C h^{k+1},
$$

where the constant $C$ is independent of $h$ and depends on the terminal time $T$ and the exact solution $u$.

Proof. Note that the scheme $(5.3)$ is also satisfied when the numerical solution $\left(u_{h}(\cdot), v_{h}(\cdot)\right)$ is replaced with the exact solution $(u(\cdot), v(\cdot))$ : for any $(\omega, t) \in \Omega \times[0, T]$ and $r_{h}, z_{h} \in V_{h}$, we have

$$
\begin{aligned}
\int_{I_{j}} r_{h}(x) \mathrm{d} u(\cdot, x, t) \mathrm{d} x= & a H_{j}^{+}\left(v(\cdot, \cdot, t), r_{h}\right) \mathrm{d} t+\int_{I_{j}} \psi(\cdot, x, t, u(\cdot, x, t), v(\cdot, x, t)) r_{h}(x) \mathrm{d} x \mathrm{~d} t \\
& +\int_{I_{j}} g(\cdot, x, t, u(\cdot, x, t), v(\cdot, x, t)) r_{h}(x) \mathrm{d} x \mathrm{~d} W_{t}, \\
\int_{I_{j}} v(\omega, x, t) z_{h}(x) \mathrm{d} x= & H_{j}^{-}\left(u(\omega, \cdot, t), z_{h}\right) .
\end{aligned}
$$


Define

$$
e_{u}(\omega, x, t):=\left(u-u_{h}\right)(\omega, x, t)=\left(\xi_{u}-\eta_{u}\right)(\omega, x, t)
$$

and

$$
e_{v}(\omega, x, t):=\left(v-v_{h}\right)(\omega, x, t)=\left(\xi_{v}-\eta_{v}\right)(\omega, x, t)
$$

with

$$
\xi_{u}(\omega, x, t):=\left(\mathcal{Q} u-u_{h}\right)(\omega, x, t), \quad \eta_{u}(\omega, x, t):=(\mathcal{Q} u-u)(\omega, x, t)
$$

and

$$
\xi_{v}(\omega, x, t):=\left(\mathcal{R} v-v_{h}\right)(\omega, x, t), \quad \eta_{v}(\omega, x, t):=(\mathcal{R} v-v)(\omega, x, t),
$$

where $\mathcal{R}$ and $\mathcal{Q}$ are the projections from $H^{k+1}$ onto $V_{h}$ defined in Section 2.

Then the error equation is

$$
\begin{aligned}
\int_{I_{j}} r_{h}(x) d e_{u}(x, t) \mathrm{d} x= & \left\{a H_{j}^{+}\left(e_{v}(\cdot, t), r_{h}\right)+\int_{I_{j}}\left[\psi(\cdot, u, v)-\psi\left(\cdot, u_{h}, v_{h}\right)\right](x, t) r_{h}(x) \mathrm{d} x\right\} \mathrm{d} t \\
& +\int_{I_{j}}\left[g(x, t, u(x, t), v(x, t))-g\left(x, t, u_{h}(x, t), v_{h}(x, t)\right)\right] r_{h}(x) \mathrm{d} x \mathrm{~d} W_{t}, \\
& \int_{I_{j}} e_{v}(x, t) z_{h}(x) \mathrm{d} x=H_{j}^{-}\left(e_{u}(\cdot, t), z_{h}\right) .
\end{aligned}
$$

Taking $r_{h}=\xi_{u}(\omega, \cdot, t)$ in $(5.4 \mathrm{a})$ and $z_{h}=a \xi_{v}(\omega, \cdot, t)$ in $(5.4 \mathrm{~b})$, we have

$$
\begin{aligned}
\int_{I_{j}} \xi_{u}(x, t) d \xi_{u}(x, t) \mathrm{d} x+ & a \int_{I_{j}}\left|\xi_{v}(x, t)\right|^{2} \mathrm{~d} x \mathrm{~d} t \\
= & \int_{I_{j}} \xi_{u}(x, t) d \eta_{u}(x, t) \mathrm{d} x+a \int_{I_{j}} \eta_{v}(x, t) \xi_{v}(x, t) \mathrm{d} x \mathrm{~d} t \\
& +a\left[H_{j}^{+}\left(\xi_{v}(\cdot, t), \xi_{u}(\cdot, t)\right)-H_{j}^{+}\left(\eta_{v}(\cdot, t), \xi_{u}(\cdot, t)\right)\right] \mathrm{d} t \\
& +\int_{I_{j}}\left[\psi(x, t, u(x, t), v(x, t))-\psi\left(x, t, u_{h}(x, t), v_{h}(x, t)\right)\right] \xi_{u}(x, t) \mathrm{d} x \mathrm{~d} t \\
& +a\left[H_{j}^{-}\left(\xi_{u}(\cdot, t), \xi_{v}(\cdot, t)\right)-H_{j}^{-}\left(\eta_{u}(\cdot, t), \xi_{v}(\cdot, t)\right)\right] \mathrm{d} t \\
& +\int_{I_{j}}\left[g(x, t, u(x, t), v(x, t))-g\left(x, t, u_{h}(x, t), v_{h}(x, t)\right)\right] \xi_{u}(x, t) \mathrm{d} x \mathrm{~d} W_{t} .
\end{aligned}
$$

Using the Itô's formula, we have

$$
d\left|\xi_{u}(x, t)\right|^{2}=2 \xi_{u}(x, t) d \xi_{u}(x, t)+d\left\langle\xi_{u}(x, \cdot), \xi_{u}(x, \cdot)\right\rangle_{t} .
$$

Then, we have

$$
\begin{aligned}
\mathbb{E}\left[\left\|\xi_{u}(\cdot, t)\right\|^{2}\right] & +2 a \mathbb{E}\left[\int_{0}^{t} \int_{0}^{2 \pi}\left|\xi_{v}(x, s)\right|^{2} \mathrm{~d} x \mathrm{~d} s\right] \\
& =\left\|\xi_{u}(\cdot, 0)\right\|^{2}+\mathcal{T}_{1}(t)+\mathcal{T}_{2}(t)+\mathcal{T}_{3}(t)+\mathcal{T}_{4}(t)+\mathcal{T}_{5}(t)+\mathcal{T}_{6}(t)+\mathcal{T}_{7}(t)
\end{aligned}
$$


where

$$
\begin{aligned}
& \mathcal{T}_{1}(t):=2 \mathbb{E}\left[\int_{0}^{2 \pi} \int_{0}^{t} \xi_{u}(x, s) d \eta_{u}(x, s) \mathrm{d} x\right], \\
& \mathcal{T}_{2}(t):=\mathbb{E}\left[\int_{0}^{2 \pi}\left\langle\xi_{u}(x, \cdot), \xi_{u}(x, \cdot)\right\rangle_{t} \mathrm{~d} x\right], \\
& \mathcal{T}_{3}(t):=2 a \mathbb{E}\left[\int_{0}^{t} \int_{0}^{2 \pi} \eta_{v}(x, s) \xi_{v}(x, s) \mathrm{d} x \mathrm{~d} s\right], \\
& \mathcal{T}_{4}(t):=2 a \mathbb{E}\left[\int_{0}^{t} \sum_{j=1}^{N}\left[H_{j}^{+}\left(\xi_{v}(\cdot, s), \xi_{u}(\cdot, s)\right)+H_{j}^{-}\left(\xi_{u}(\cdot, s), \xi_{v}(\cdot, s)\right)\right] \mathrm{d} s\right], \\
& \mathcal{T}_{5}(t):=-2 a \mathbb{E}\left[\int_{0}^{t} \sum_{j=1}^{N}\left[H_{j}^{+}\left(\eta_{v}(\cdot, s), \xi_{u}(\cdot, s)\right)+H_{j}^{-}\left(\eta_{u}(\cdot, s), \xi_{v}(\cdot, s)\right)\right] \mathrm{d} s\right], \\
& \mathcal{T}_{6}(t):=2 \mathbb{E}\left[\int_{0}^{t} \int_{0}^{2 \pi}\left[\psi(x, s, u(x, s), v(x, s))-\psi\left(x, s, u_{h}(x, s), v_{h}(x, s)\right)\right] \xi_{u}(x, s) \mathrm{d} x \mathrm{~d} s\right],
\end{aligned}
$$

and

$$
\mathcal{T}_{7}(t):=2 \mathbb{E}\left[\int_{0}^{t} \int_{0}^{2 \pi}\left[g(x, s, u(x, s), v(x, s))-g\left(x, s, u_{h}(x, s), v_{h}(x, s)\right)\right] \xi_{u}(x, s) \mathrm{d} x \mathrm{~d} W_{s}\right] .
$$

The terms $\mathcal{T}_{i}(t)$ for $i=1, \ldots, 7$ are estimated as follows.

- The estimate of $\mathcal{T}_{1}(t)$.

In view of (5.1), we have

$$
\begin{aligned}
d_{t}(\mathcal{Q} u)(\cdot, t) & =\mathcal{Q}\left(d_{t} u\right)(\cdot, t) \\
& =\mathcal{Q}\left[a u_{x x}(\cdot, t)\right] \mathrm{d} t+\mathcal{Q}\left[\psi\left(\cdot, t, u(\cdot, t), u_{x}(\cdot, t)\right)\right] \mathrm{d} t+\mathcal{Q}\left[g\left(\cdot, t, u(\cdot, t), u_{x}(\cdot, t)\right)\right] \mathrm{d} W_{t}
\end{aligned}
$$

Therefore,

$$
\begin{aligned}
d \eta_{u}(\cdot, t)= & a\left(\mathcal{Q} u_{x x}-u_{x x}\right)(\cdot, t) \mathrm{d} t+(\mathcal{Q}-\mathcal{I}) \psi\left(\cdot, t, u(\cdot, t), u_{x}(\cdot, t)\right) \mathrm{d} t \\
& +(\mathcal{Q}-\mathcal{I}) g\left(\cdot, t, u(\cdot, t), u_{x}(\cdot, t)\right) \mathrm{d} W_{t}
\end{aligned}
$$

with $\mathcal{I}$ being the identity operator.

It turns out that

$$
\begin{aligned}
\int_{0}^{2 \pi} \xi_{u}(x, t) d \eta_{u}(x, t) \mathrm{d} x= & \int_{0}^{2 \pi} \xi_{u}(x, t)\left\{a\left[\mathcal{Q} u_{x x}-u_{x x}\right](x, t)+(\mathcal{Q}-\mathcal{I})\left[\psi\left(\cdot, t, u(\cdot, t), u_{x}(\cdot, t)\right)\right](x)\right\} \mathrm{d} x \mathrm{~d} t \\
& +\int_{0}^{2 \pi} \xi_{u}(x, t)(\mathcal{Q}-\mathcal{I})\left[g\left(\cdot, t, u(\cdot, t), u_{x}(\cdot, t)\right)\right](x) \mathrm{d} x \mathrm{~d} W_{t} .
\end{aligned}
$$

According to Lemma 2.1 , by virtue of $(3.17), g\left(\cdot, u(\cdot), u_{x}(\cdot)\right) \in L^{2}\left(\Omega \times[0, T] ; L^{2}\right)$ and $u(\cdot) \quad \in$ $\mathcal{S}^{2}\left(\Omega \times[0, T] ; L^{2}\right)$, we get that the process

$$
\int_{0}^{t} \int_{0}^{2 \pi} \xi_{u}(x, s)(\mathcal{Q}-\mathcal{I})\left[g\left(\cdot, s, u(\cdot, s), u_{x}(\cdot, s)\right)\right](x) \mathrm{d} x \mathrm{~d} W_{s}, \quad 0 \leq t \leq T
$$


is a martingale. Thus according to the property of the projection (2.3), we have

$$
\begin{aligned}
\mathcal{T}_{1}(t)= & 2 a \mathbb{E}\left[\int_{0}^{t} \int_{0}^{2 \pi} \xi_{u}(x, s)\left[\mathcal{Q} u_{x x}-u_{x x}\right](x, s) \mathrm{d} x \mathrm{~d} s\right] \\
& +2 \mathbb{E}\left[\int_{0}^{t} \int_{0}^{2 \pi} \xi_{u}(x, s)(\mathcal{Q}-\mathcal{I})\left[\psi\left(\cdot, s, u(\cdot, s), u_{x}(\cdot, s)\right)\right](x) \mathrm{d} x \mathrm{~d} s\right] \\
\leq & a \mathbb{E}\left[\int_{0}^{t}\left(\left\|\xi_{u}(\cdot, s)\right\|^{2}+\left\|\left(\mathcal{Q} u_{x x}-u_{x x}\right)(\cdot, s)\right\|^{2}\right) \mathrm{d} s\right] \\
& +\mathbb{E}\left[\int_{0}^{t}\left(\left\|\xi_{u}(\cdot, s)\right\|^{2}+\left\|(\mathcal{Q}-\mathcal{I}) \psi\left(\cdot, s, u(\cdot, s), u_{x}(\cdot, s)\right)\right\|^{2}\right) \mathrm{d} s\right] \\
\leq & C \int_{0}^{t} \mathbb{E}\left\|\xi_{u}(\cdot, s)\right\|^{2} \mathrm{~d} s+C h^{2 k+2} \mathbb{E}\left[\int_{0}^{t}\left\|u_{x x}(\cdot, s)\right\|_{H^{k+1}}^{2} \mathrm{~d} s\right] \\
& +C h^{2 k+2} \mathbb{E}\left[\int_{0}^{t}\left\|\psi\left(\cdot, s, u(\cdot, s), u_{x}(\cdot, s)\right)\right\|_{H^{k+1}}^{2} \mathrm{~d} s\right] .
\end{aligned}
$$

Since

$$
u \in L^{2}\left(\Omega \times[0, T] ; H^{k+3}\right), \quad \psi\left(\cdot, u(\cdot), u_{x}(\cdot)\right) \in L^{2}\left(\Omega \times[0, T] ; H^{k+1}\right),
$$

we have

$$
\begin{aligned}
\mathcal{T}_{1}(t) \leq & C \int_{0}^{t} \mathbb{E}\left\|\xi_{u}(\cdot, s)\right\|^{2} \mathrm{~d} s+C h^{2 k+2} \mathbb{E}\left[\int_{0}^{t}\|u(\cdot, s)\|_{H^{k+3}}^{2} \mathrm{~d} s\right] \\
& +C h^{2 k+2} \mathbb{E}\left[\int_{0}^{t}\left\|\psi\left(\cdot, s, u(\cdot, s), u_{x}(\cdot, s)\right)\right\|_{H^{k+1}}^{2} \mathrm{~d} s\right] \\
\leq & C \int_{0}^{t} \mathbb{E}\left\|\xi_{u}(\cdot, s)\right\|^{2} \mathrm{~d} s+C h^{2 k+2} .
\end{aligned}
$$

- The estimate of $\mathcal{T}_{2}(t)$.

In view of (5.5), we have that for any $r_{h} \in V_{h}$,

$$
\begin{aligned}
\int_{I_{j}} r_{h}(x) d \mathcal{Q} u(x, t) \mathrm{d} x= & \int_{I_{j}} r_{h}(x)\left\{\mathcal{Q}\left[a u_{x x}(\cdot, t)\right](x)+\mathcal{Q}[\psi(\cdot, t, u(\cdot, t), v(\cdot, t))](x)\right\} \mathrm{d} x \mathrm{~d} t \\
& +\int_{I_{j}} r_{h}(x) \mathcal{Q}[g(\cdot, t, u(\cdot, t), v(\cdot, t))](x) \mathrm{d} x \mathrm{~d} W_{t} .
\end{aligned}
$$

From (5.3a) and (5.6), we obtain that for any $r_{h} \in V_{h}$,

$$
\begin{aligned}
\int_{I_{j}} r_{h}(x) d \xi_{u}(x, t) \mathrm{d} x= & \left\{a \int_{I_{j}} r_{h}(x) \mathcal{Q}\left[u_{x x}(\cdot, t)\right](x) \mathrm{d} x-a H_{j}^{+}\left(v_{h}(\cdot, t), r_{h}\right)\right. \\
& \left.+\int_{I_{j}} r_{h}(x)\left\{\mathcal{Q}[\psi(\cdot, t, u(\cdot, t), v(\cdot, t))]-\psi\left(\cdot, t, u_{h}(\cdot, t), v_{h}(\cdot, t)\right)\right\}(x) \mathrm{d} x\right\} \mathrm{d} t \\
& +\int_{I_{j}} r_{h}(x)\left\{\mathcal{Q}[g(\cdot, t, u(\cdot, t), v(\cdot, t))]-g\left(\cdot, t, u_{h}(\cdot, t), v_{h}(\cdot, t)\right)\right\}(x) \mathrm{d} x \mathrm{~d} W_{t} .
\end{aligned}
$$

Since $\xi_{u}(\omega, \cdot, t) \in V_{h}$ for any $(\omega, t) \in \Omega \times[0, T], \xi_{u}$ should have the form

$$
\xi_{u}(\omega, x, t)=\sum_{l=0}^{k} \xi_{l, j}^{u}(\omega, t) \varphi_{l}^{j}(x), \quad x \in I_{j} .
$$


Similar to (4.4), we have from (5.7) that

$$
\begin{aligned}
\int_{I_{j}}\left\langle\xi_{u}(x, \cdot), \xi_{u}(x, \cdot)\right\rangle_{t} \mathrm{~d} x= & \int_{0}^{t} \int_{I_{j}}\left(\mathcal{P}\left\{\mathcal{Q}[g(\cdot, s, u(\cdot, s), v(\cdot, s))]-g\left(\cdot, s, u_{h}(\cdot, s), v_{h}(\cdot, s)\right)\right\}(x)\right. \\
& \left.\times\left\{\mathcal{Q}[g(\cdot, s, u(\cdot, s), v(\cdot, s))]-g\left(\cdot, s, u_{h}(\cdot, s), v_{h}(\cdot, s)\right)\right\}(x)\right) \mathrm{d} x \mathrm{~d} s \\
\leq & \int_{0}^{t} \int_{I_{j}}\left|\mathcal{Q}[g(\cdot, s, u(\cdot, s), v(\cdot, s))]-g\left(\cdot, s, u_{h}(\cdot, s), v_{h}(\cdot, s)\right)\right|^{2}(x) \mathrm{d} x \mathrm{~d} s .
\end{aligned}
$$

Then, we have for any $\varepsilon>0$,

$$
\begin{aligned}
\mathcal{T}_{2}(t)= & \mathbb{E}\left[\int_{0}^{2 \pi}\left\langle\xi_{u}(x, \cdot), \xi_{u}(x, \cdot)\right\rangle_{t} \mathrm{~d} x\right] \\
\leq & \mathbb{E}\left[\int_{0}^{t} \int_{0}^{2 \pi}\left|\mathcal{Q}[g(\cdot, s, u(\cdot, s), v(\cdot, s))]-g\left(\cdot, s, u_{h}(\cdot, s), v_{h}(\cdot, s)\right)\right|^{2}(x) \mathrm{d} x \mathrm{~d} s\right] \\
\leq & \left(1+\frac{1}{\varepsilon}\right) \mathbb{E}\left[\int_{0}^{t} \int_{0}^{2 \pi}|(\mathcal{Q}-\mathcal{I}) g(\cdot, s, u(\cdot, s), v(\cdot, s))|^{2}(x) \mathrm{d} x \mathrm{~d} s\right] \\
& +(1+\varepsilon) \mathbb{E}\left[\int_{0}^{t} \int_{0}^{2 \pi}\left|g(x, s, u(x, s), v(x, s))-g\left(x, s, u_{h}(x, s), v_{h}(x, s)\right)\right|^{2} \mathrm{~d} x \mathrm{~d} s\right] .
\end{aligned}
$$

According to (H4) and the property of the projection, we have

$$
\begin{aligned}
\mathcal{T}_{2}(t) \leq & \left(1+\frac{1}{\varepsilon}\right) h^{2 k+2} \mathbb{E}\left[\int_{0}^{t}\|g(\cdot, s, u(\cdot, s), v(\cdot, s))\|_{H^{k+1}}^{2} \mathrm{~d} s\right] \\
& +(1+\varepsilon) \mathbb{E} \int_{0}^{t} \int_{0}^{2 \pi}\left[C_{1}^{2}\left(1+\frac{1}{\varepsilon}\right)\left|e_{u}(x, s)\right|^{2}+C_{2}^{2}(1+\varepsilon)\left|e_{v}(x, s)\right|^{2}\right] \mathrm{d} x \mathrm{~d} s \\
\leq & \left(1+\frac{1}{\varepsilon}\right) h^{2 k+2} \mathbb{E}\left[\int_{0}^{t}\|g(\cdot, s, u(\cdot, s), v(\cdot, s))\|_{H^{k+1}}^{2} \mathrm{~d} s\right] \\
& +2(1+\varepsilon)\left(1+\frac{1}{\varepsilon}\right) C_{1}^{2} \mathbb{E} \int_{0}^{t}\left[C h^{2 k+2}\|u(\cdot, s)\|_{H^{k+1}}^{2}+\left\|\xi_{u}(\cdot, s)\right\|^{2}\right] \mathrm{d} s \\
& +(1+\varepsilon)^{2}\left(1+\frac{1}{\varepsilon}\right) C_{2}^{2} \mathbb{E}\left[\int_{0}^{t} C h^{2 k+2}\|v(\cdot, s)\|_{H^{k+1}}^{2} \mathrm{~d} s\right] \\
& +(1+\varepsilon)^{3} C_{2}^{2} \mathbb{E}\left[\int_{0}^{t}\left\|\xi_{v}(\cdot, s)\right\|^{2} \mathrm{~d} s\right] .
\end{aligned}
$$

Since $u \in L^{2}\left(\Omega \times[0, T] ; H^{k+3}\right)$, we have

$$
v=u_{x} \in L^{2}\left(\Omega \times[0, T] ; H^{k+2}\right) \subseteq L^{2}\left(\Omega \times[0, T] ; H^{k+1}\right),
$$

which yields that

$$
\begin{aligned}
\mathcal{T}_{2}(t) \leq & C\left(1+\frac{1}{\varepsilon}\right)\left[1+(1+\varepsilon)+(1+\varepsilon)^{2}\right] h^{2 k+2} \\
& +C(1+\varepsilon)\left(1+\frac{1}{\varepsilon}\right) \int_{0}^{t} \mathbb{E}\left[\left\|\xi_{u}(\cdot, s)\right\|^{2}\right] \mathrm{d} s+(1+\varepsilon)^{3} C_{2}^{2} \mathbb{E}\left[\int_{0}^{t}\left\|\xi_{v}(\cdot, s)\right\|^{2} \mathrm{~d} s\right] .
\end{aligned}
$$

Since $2 a>C_{2}^{2}$, taking

$$
\varepsilon:=\left(\frac{2 a+C_{2}^{2}}{2 C_{2}^{2}}\right)^{\frac{1}{3}}-1>0,
$$


we have

$$
\mathcal{T}_{2}(t) \leq C h^{2 k+2}+C \int_{0}^{t} \mathbb{E}\left[\left\|\xi_{u}(\cdot, s)\right\|^{2}\right] \mathrm{d} s+\left(a+\frac{1}{2} C_{2}^{2}\right) \mathbb{E}\left[\int_{0}^{t}\left\|\xi_{v}(\cdot, s)\right\|^{2} \mathrm{~d} s\right]
$$

- The estimate of $\mathcal{T}_{3}(t)$.

Since $v \in L^{2}\left(\Omega \times[0, T] ; H^{k+1}\right)$, we have

$$
\begin{aligned}
\mathcal{T}_{3}(t) & =2 a \mathbb{E}\left[\int_{0}^{t} \int_{0}^{2 \pi} \eta_{v}(x, s) \xi_{v}(x, s) \mathrm{d} x \mathrm{~d} s\right] \\
& \leq \frac{4 a^{2}}{2 a-C_{2}^{2}} \mathbb{E}\left[\int_{0}^{t}\left\|\eta_{v}(\cdot, s)\right\|^{2} \mathrm{~d} s\right]+\frac{2 a-C_{2}^{2}}{4} \mathbb{E}\left[\int_{0}^{t}\left\|\xi_{v}(\cdot, s)\right\|^{2} \mathrm{~d} s\right] \\
& \leq \frac{4 a^{2}}{2 a-C_{2}^{2}} C h^{2 k+2} \mathbb{E}\left[\int_{0}^{t}\|v(\cdot, s)\|_{H^{k+1}}^{2} \mathrm{~d} s\right]+\frac{2 a-C_{2}^{2}}{4} \mathbb{E}\left[\int_{0}^{t}\left\|\xi_{v}(\cdot, s)\right\|^{2} \mathrm{~d} s\right] \\
& \leq C h^{2 k+2}+\frac{2 a-C_{2}^{2}}{4} \mathbb{E}\left[\int_{0}^{t}\left\|\xi_{v}(\cdot, s)\right\|^{2} \mathrm{~d} s\right] .
\end{aligned}
$$

- The estimate of $\mathcal{T}_{4}(t)$.

In view of (4.5), we see that for any $u, v \in V_{h}$,

$$
\sum_{j=1}^{N}\left[H_{j}^{+}(u, v)+H_{j}^{-}(v, u)\right]=0 .
$$

Therefore,

$$
\mathcal{T}_{4}(t)=2 a \mathbb{E}\left[\int_{0}^{t} \sum_{j=1}^{N}\left[H_{j}^{+}\left(\xi_{v}(\cdot, s), \xi_{u}(\cdot, s)\right)+H_{j}^{-}\left(\xi_{u}(\cdot, s), \xi_{v}(\cdot, s)\right)\right] \mathrm{d} s\right]=0 .
$$

- The estimate of $\mathcal{T}_{5}(t)$.

By the definition of the projections $\mathcal{Q}$ and $\mathcal{R}$ (see (2.1) and (2.2)), we see that for any $(\omega, s) \in \Omega \times[0, T]$, $j=1,2, \ldots, N$, and $r_{h}, z_{h} \in V_{h}$,

$$
H_{j}^{-}\left(\eta_{u}(\cdot, s), r_{h}\right)=0, \quad H_{j}^{+}\left(\eta_{v}(\cdot, s), z_{h}\right)=0 .
$$

Since $\xi_{u}(\omega, \cdot, s), \xi_{v}(\omega, \cdot, s) \in V_{h}$, we have

$$
\mathcal{T}_{5}(t)=-2 a \mathbb{E}\left[\int_{0}^{t} \sum_{j=1}^{N}\left[H_{j}^{+}\left(\eta_{v}(\cdot, s), \xi_{u}(\cdot, s)\right)+H_{j}^{-}\left(\eta_{u}(\cdot, s), \xi_{v}(\cdot, s)\right)\right] \mathrm{d} s\right]=0 .
$$

- The estimate of $\mathcal{T}_{6}(t)$. 
We have from (H3) and Young's inequality that

$$
\begin{aligned}
\mathcal{T}_{6}(t)= & 2 \mathbb{E}\left[\int_{0}^{t} \int_{0}^{2 \pi}\left[\psi(x, s, u(x, s), v(x, s))-\psi\left(x, s, u_{h}(x, s), v_{h}(x, s)\right)\right] \xi_{u}(x, s) \mathrm{d} x \mathrm{~d} s\right] \\
\leq & 2 B_{1} \mathbb{E}\left[\int_{0}^{t} \int_{0}^{2 \pi}\left(\left|\xi_{u}(x, s)\right|+\left|\eta_{u}(x, s)\right|+\left|\xi_{v}(x, s)\right|+\left|\eta_{v}(x, s)\right|\right)\left|\xi_{u}(x, s)\right| \mathrm{d} x \mathrm{~d} s\right] \\
\leq & B_{1} \mathbb{E}\left[\int_{0}^{t} \int_{0}^{2 \pi}\left[\left|\eta_{u}(x, s)\right|^{2}+\left|\eta_{v}(x, s)\right|^{2}\right] \mathrm{d} x \mathrm{~d} s\right] \\
& +B_{1} \mathbb{E}\left[\int_{0}^{t} \int_{0}^{2 \pi}\left[\left(4+\frac{8 B_{1}}{2 a-C_{2}^{2}}\right)\left|\xi_{u}(x, s)\right|^{2}+\frac{2 a-C_{2}^{2}}{8 B_{1}}\left|\xi_{v}(x, s)\right|^{2}\right] \mathrm{d} x \mathrm{~d} s\right] \\
\leq & C \mathbb{E} \int_{0}^{t}\left[\left\|\eta_{u}(\cdot, s)\right\|^{2}+\left\|\eta_{v}(\cdot, s)\right\|^{2}+\left\|\xi_{u}(\cdot, s)\right\|^{2}\right] \mathrm{d} s+\frac{2 a-C_{2}^{2}}{8} \mathbb{E}\left[\int_{0}^{t}\left\|\xi_{v}(\cdot, s)\right\|^{2} \mathrm{~d} s\right] .
\end{aligned}
$$

Using the property of projection (2.3), we have

$$
\begin{aligned}
\mathcal{T}_{6}(t) \leq & C h^{2 k+2} \mathbb{E}\left[\int_{0}^{t}\|u(\cdot, s)\|_{H^{k+1}}^{2} \mathrm{~d} s\right]+C h^{2 k+2} \mathbb{E}\left[\int_{0}^{t}\|v(\cdot, s)\|_{H^{k+1}}^{2} \mathrm{~d} s\right] \\
& +C \mathbb{E}\left[\int_{0}^{t}\left\|\xi_{u}(\cdot, s)\right\|^{2} \mathrm{~d} s\right]+\frac{2 a-C_{2}^{2}}{8} \mathbb{E}\left[\int_{0}^{t}\left\|\xi_{v}(\cdot, s)\right\|^{2} \mathrm{~d} s\right] .
\end{aligned}
$$

Therefore,

$$
\mathcal{T}_{6}(t) \leq C h^{2 k+2}+C \int_{0}^{t} \mathbb{E}\left[\left\|\xi_{u}(\cdot, s)\right\|^{2}\right] \mathrm{d} s+\frac{2 a-C_{2}^{2}}{8} \mathbb{E}\left[\int_{0}^{t}\left\|\xi_{v}(\cdot, s)\right\|^{2} \mathrm{~d} s\right] .
$$

- The estimate of $\mathcal{T}_{7}(t)$.

According to Lemma 2.1, by virtue of (3.17), (3.18), $g\left(\cdot, u(\cdot), u_{x}(\cdot)\right) \in L^{2}\left(\Omega \times[0, T] ; L^{2}\right)$ and $u(\cdot) \in$ $\mathcal{S}^{2}\left(\Omega \times[0, T] ; L^{2}\right)$, we see that the process

$$
\left.\int_{0}^{t} \int_{0}^{2 \pi}[g(x, s, u(x, s), v(x, s)))-g\left(x, s, u_{h}(x, s), v_{h}(x, s)\right)\right] \xi_{u}(x, s) \mathrm{d} x \mathrm{~d} W_{s}, \quad 0 \leq t \leq T
$$

is a martingale. Thus,

$$
\mathcal{T}_{7}(t)=2 \mathbb{E}\left[\int_{0}^{t} \int_{0}^{2 \pi}\left[g(x, s, u(x, s), v(x, s))-g\left(x, s, u_{h}(x, s), v_{h}(x, s)\right)\right] \xi_{u}(x, s) \mathrm{d} x \mathrm{~d} W_{s}\right]=0 .
$$

Concluding the above, we have

$$
\begin{aligned}
\mathbb{E}\left[\left\|\xi_{u}(\cdot, t)\right\|^{2}\right] & +\frac{2 a-C_{2}^{2}}{8} \mathbb{E}\left[\int_{0}^{t} \int_{0}^{2 \pi}\left|\xi_{v}(x, s)\right|^{2} \mathrm{~d} x \mathrm{~d} s\right] \\
& \leq\left\|\xi_{u}(\cdot, 0)\right\|^{2}+C h^{2 k+2}+C \int_{0}^{t} \mathbb{E}\left[\left\|\xi_{u}(\cdot, s)\right\|^{2}\right] \mathrm{d} s .
\end{aligned}
$$

Since $\left\|\xi_{u}(\cdot, 0)\right\|=\left\|\mathcal{Q} u_{0}-\mathcal{P} u_{0}\right\| \leq C h^{k+1}\left\|u_{0}\right\|_{H^{k+1}}$, we have from Gronwall's inequality that

$$
\left(\mathbb{E}\left[\left\|\xi_{u}(\cdot, t)\right\|^{2}\right]\right)^{\frac{1}{2}} \leq C h^{k+1} e^{C t},
$$


which yields

$$
\left(\mathbb{E}\left[\int_{0}^{t} \int_{0}^{2 \pi}\left|\xi_{v}(x, s)\right|^{2} \mathrm{~d} x \mathrm{~d} s\right]\right)^{\frac{1}{2}} \leq C h^{k+1} e^{C t} .
$$

Since $u \in L^{\infty}\left(0, T ; L^{2}\left(\Omega ; H^{k+1}\right)\right)$, we have

$$
\left(\mathbb{E}\left[\left\|\eta_{u}(\cdot, t)\right\|^{2}\right]\right)^{\frac{1}{2}} \leq C\left(\mathbb{E}\left[\|u(\cdot, t)\|_{H^{k+1}}^{2}\right]\right)^{\frac{1}{2}} h^{k+1} \leq C h^{k+1} .
$$

It turns out that

$$
\left(\mathbb{E}\left[\left\|u(\cdot, t)-u_{h}(\cdot, t)\right\|^{2}\right]\right)^{\frac{1}{2}} \leq\left(\mathbb{E}\left[\left\|\xi_{u}(\cdot, t)\right\|^{2}\right]\right)^{\frac{1}{2}}+\left(\mathbb{E}\left[\left\|\eta_{u}(\cdot, t)\right\|^{2}\right]\right)^{\frac{1}{2}} \leq C e^{C t} h^{k+1} .
$$

Since $v \in L^{2}\left(\Omega \times[0, T] ; H^{k+1}\right)$, we have

$$
\left(\mathbb{E}\left[\int_{0}^{t} \int_{0}^{2 \pi}\left|\eta_{v}(x, s)\right|^{2} \mathrm{~d} x \mathrm{~d} s\right]\right)^{\frac{1}{2}} \leq C\left(\mathbb{E}\left[\int_{0}^{t}\|v(\cdot, s)\|_{H^{k+1}}^{2} \mathrm{~d} s\right]\right)^{\frac{1}{2}} h^{k+1} \leq C h^{k+1} .
$$

Thus, we have

$$
\begin{aligned}
& \left(\mathbb{E}\left[\int_{0}^{t}\left\|u_{x}(\cdot, s)-v_{h}(\cdot, s)\right\|^{2} \mathrm{~d} s\right]\right)^{\frac{1}{2}} \\
\leq & \left(\mathbb{E}\left[\int_{0}^{t} \int_{0}^{2 \pi}\left|\eta_{v}(x, s)\right|^{2} \mathrm{~d} x \mathrm{~d} s\right]\right)^{\frac{1}{2}}+\left(\mathbb{E}\left[\int_{0}^{t} \int_{0}^{2 \pi}\left|\xi_{v}(x, s)\right|^{2} \mathrm{~d} x \mathrm{~d} s\right]\right)^{\frac{1}{2}} \leq C h^{k+1} e^{C t} .
\end{aligned}
$$

Remark 5.2. The error estimate is optimal regarding the polynomial degree $k$ but may be not optimal regarding the required high-regularity of the exact solution $u$ in assumption (H5), whose integrability and differentiability are used to derive our error estimate. Note that the high regularities required in (H5) can be found in $[18,24]$ for some special SPDEs (1.1), like the linear SPDEs (1.1) in [18] and the semilinear SPDEs (1.1) in [24] where $a(\cdot)$ does not depend on $\left(u, u_{x}\right)$ and $g(\cdot)$ does not depend on $u_{x}$.

\section{Time DiscRetizATion}

The LDG method incorporates the spatial discretization and reduces the primal SPDE into a system of SDEs, which needs to be coupled with a high-order time discretization. We will propose a numerical scheme which avoids the usage of derivatives in much the same way that Runge-Kutta schemes do in the deterministic setting. For notational simplicity, we shall mainly state the schemes for the autonomous case. Consider the following matrix-valued SDE:

$$
\left\{\begin{aligned}
d X_{t}^{i, j} & =a^{i, j}\left(X_{t}\right) \mathrm{d} t+b^{i, j}\left(X_{t}\right) \mathrm{d} W_{t}, \quad t>0 \\
X_{0}^{i, j} & =x_{0}^{i, j}
\end{aligned}\right.
$$

where $i=0,1, \ldots, k$ and $j=0,1, \ldots, N+1$. We aim to use $Y_{n}^{i, j}$ to approximate $X_{t_{n}}^{i, j}$. Define $Y_{0}^{i, j}:=x_{0}^{i, j}$. Suppose we already have $\left\{Y_{n}^{i, j}: i=0,1, \ldots, k\right.$ and $\left.j=0,1, \ldots, N+1\right\}$.

We use an explicit derivative-free strong scheme of order 1.5 from Kloeden and Platen [27]. For convenience of the reader, we give a detailed description here. 
Define

$$
\Delta_{n}:=t_{n+1}-t_{n}, \quad \Delta W_{n}:=W_{t_{n+1}}-W_{t_{n}}, \quad \Delta Z_{n}:=\int_{t_{n}}^{t_{n+1}}\left(W_{s}-W_{t_{n}}\right) \mathrm{d} s .
$$

We set

$$
\gamma_{ \pm}^{m, l}:=Y_{n}^{m, l}+a^{m, l}\left(Y_{n}\right) \Delta_{n} \pm b^{m, l}\left(Y_{n}\right) \sqrt{\Delta_{n}}
$$

and

$$
\phi_{ \pm}^{m, l}:=\gamma_{+}^{m, l} \pm b^{m, l}\left(\gamma_{+}\right) \sqrt{\Delta_{n}}
$$

Then, an explicit order 1.5 strong scheme has the form

$$
\begin{aligned}
Y_{n+1}^{i, j}= & Y_{n}^{i, j}+a^{i, j}\left(Y_{n}\right) \Delta_{n}+b^{i, j}\left(Y_{n}\right) \Delta W_{n} \\
& (\text { order } 0.5) \\
+ & \frac{1}{4 \sqrt{\Delta_{n}}}\left\{b^{i, j}\left(\gamma_{+}\right)-b^{i, j}\left(\gamma_{-}\right)\right\}\left\{\left(\Delta W_{n}\right)^{2}-\Delta_{n}\right\} \\
& \quad(\text { order } 1.0) \\
+ & \frac{1}{4}\left\{a^{i, j}\left(\gamma_{+}\right)-2 a^{i, j}\left(Y_{n}\right)+a^{i, j}\left(\gamma_{-}\right)\right\} \Delta_{n} \\
+ & \frac{1}{2 \sqrt{\Delta_{n}}}\left\{a^{i, j}\left(\gamma_{+}\right)-a^{i, j}\left(\gamma_{-}\right)\right\} \Delta Z_{n} \\
+ & \frac{1}{2 \Delta_{n}}\left\{b^{i, j}\left(\gamma_{+}\right)-2 b^{i, j}\left(Y_{n}\right)+b^{i, j}\left(\gamma_{-}\right)\right\}\left\{\Delta W_{n} \Delta_{n}-\Delta Z_{n}\right\} \\
+ & \frac{1}{4 \Delta_{n}}\left\{b^{i, j}\left(\phi_{+}\right)-b^{i, j}\left(\phi_{-}\right)-b^{i, j}\left(\gamma_{+}\right)+b^{i, j}\left(\gamma_{-}\right)\right\}\left\{\frac{1}{3}\left(\Delta W_{n}\right)^{2}-\Delta_{n}\right\} \Delta W_{n} .
\end{aligned}
$$

(order 1.5)

Here, the additional random variable $\Delta Z_{n}$ is normally distributed with the following mean, variance and correlation:

$$
\mathbb{E}\left[\Delta Z_{n}\right]=0, \quad \mathbb{E}\left[\left(\Delta Z_{n}\right)^{2}\right]=\frac{1}{3} \Delta_{n}^{3}, \quad \mathbb{E}\left[\Delta W_{n} \Delta Z_{n}\right]=\frac{1}{2} \Delta_{n}^{2},
$$

respectively. We note that there is no difficulty in generating the pair of correlated normally distributed random variables $\Delta W_{n}$ and $\Delta Z_{n}$ using the transformation

$$
\Delta W_{n}=\zeta_{n, 1} \Delta_{n}^{\frac{1}{2}}, \quad \Delta Z_{n}=\frac{1}{2}\left(\zeta_{n, 1}+\frac{1}{\sqrt{3}} \zeta_{n, 2}\right) \Delta_{n}^{\frac{3}{2}}
$$

where $\zeta_{n, 1}$ and $\zeta_{n, 2}$ are independent and $N(0 ; 1)$ distributed random variables.

\section{NUMERICAL EXPERIMENTS}

In this section we consider the application of the numerical method, which we have defined in section 3 , on some model problems. Here, $M$ is the number of realizations of the stochastic approximate solutions. We use the average of $M$ realizations to approximate the mathematical expectation. The degree of the piecewise polynomial space $V_{h}$ is $k$. The positive real number $T$ is the terminal time. Since the considered problems are second order SPDEs, in all experiments, we need to adjust the time step to $\Delta t \sim(\Delta x)^{2}$ to guarantee the stability for the explicit time discretization. Moreover, by setting $\Delta t \sim(\Delta x)^{2}$, the scheme in time is effectively third-order. 
TABLE 1. Verification of optimal convergence for nondegenerate linear SPDEs (7.1) with multiplicative noise: $k=1, M=1000$.

\begin{tabular}{llllllll}
\hline \hline & & \multicolumn{2}{c}{$b=0.1$} & \multicolumn{2}{c}{$b=0.5$} & \multicolumn{2}{c}{$b=1.0$} \\
\cline { 2 - 8 }$T=0.1$ & $N$ & $L^{2}$ Error & Order & $L^{2}$ Error & Order & $L^{2}$ Error & Order \\
\hline \multirow{5}{*}{$T=0$} & $3.87 \mathrm{E}-02$ & - & $3.91 \mathrm{E}-02$ & - & $4.06 \mathrm{E}-02$ & - \\
& 10 & $9.65 \mathrm{E}-03$ & 2.01 & $9.76 \mathrm{E}-03$ & 2.00 & $1.01 \mathrm{E}-02$ & 2.01 \\
& 40 & $2.41 \mathrm{E}-03$ & 2.00 & $2.44 \mathrm{E}-03$ & 2.00 & $2.53 \mathrm{E}-03$ & 2.00 \\
& 80 & $6.03 \mathrm{E}-04$ & 2.00 & $6.10 \mathrm{E}-04$ & 2.00 & $6.35 \mathrm{E}-04$ & 2.00 \\
& 160 & $1.51 \mathrm{E}-04$ & 2.00 & $1.53 \mathrm{E}-04$ & 1.99 & $1.60 \mathrm{E}-04$ & 1.99 \\
\hline \multirow{5}{*}{$T=0.5$} & 10 & $2.60 \mathrm{E}-02$ & - & $2.76 \mathrm{E}-02$ & - & $3.36 \mathrm{E}-02$ & - \\
& 20 & $6.48 \mathrm{E}-03$ & 2.00 & $6.86 \mathrm{E}-03$ & 2.01 & $8.17 \mathrm{E}-03$ & 2.04 \\
& 40 & $1.62 \mathrm{E}-03$ & 2.00 & $1.72 \mathrm{E}-03$ & 2.00 & $2.01 \mathrm{E}-03$ & 2.02 \\
& 80 & $4.05 \mathrm{E}-04$ & 2.00 & $4.31 \mathrm{E}-04$ & 2.00 & $5.22 \mathrm{E}-04$ & 1.95 \\
& 160 & $1.01 \mathrm{E}-04$ & 2.00 & $1.09 \mathrm{E}-04$ & 1.99 & $1.30 \mathrm{E}-04$ & 2.01 \\
\hline & 10 & $1.58 \mathrm{E}-02$ & - & $1.79 \mathrm{E}-02$ & - & $2.67 \mathrm{E}-02$ & - \\
& 20 & $3.94 \mathrm{E}-03$ & 2.00 & $4.41 \mathrm{E}-03$ & 2.02 & $6.17 \mathrm{E}-03$ & 2.11 \\
& 40 & $9.85 \mathrm{E}-04$ & 2.00 & $1.10 \mathrm{E}-03$ & 2.00 & $1.46 \mathrm{E}-03$ & 2.08 \\
& 80 & $2.46 \mathrm{E}-04$ & 2.00 & $2.79 \mathrm{E}-04$ & 1.98 & $4.04 \mathrm{E}-04$ & 1.85 \\
& 160 & $6.18 \mathrm{E}-05$ & 1.99 & $7.02 \mathrm{E}-05$ & 1.99 & $9.71 \mathrm{E}-05$ & 2.06 \\
\hline
\end{tabular}

\subsection{Nondegenerate linear SPDEs}

We consider the following linear equation

$$
\begin{cases}\mathrm{d} u=u_{x x} \mathrm{~d} t+b u \mathrm{~d} W_{t} & \text { in } \Omega \times[0,2 \pi] \times(0, T] ; \\ u(x, 0)=\sin (x), & x \in[0,2 \pi] .\end{cases}
$$

The exact solution of (7.1) is

$$
u(\omega, x, t)=\sin (x) e^{b W_{t}(\omega)-\frac{1}{2} b^{2} t-t} .
$$

In both Tables 1 and 2, we show the $L^{2}$-errors for the linear equation (7.1) with $M=1000$ realizations. We see that the scheme has $(k+1)$-th order of accuracy. The $L^{2}$-error increases as the stochastic coefficient $b$ increases. All the numerical results coincide with the conclusion of Theorem 5.1.

\subsection{Linear SPDEs with derivative in the diffusion term}

In the following we test the accuracy of the LDG method on the linear equation with first order spatial derivative involved in the diffusion term as follows,

$$
\begin{cases}\mathrm{d} u=\frac{1}{2} u_{x x} \mathrm{~d} t+b u_{x} \mathrm{~d} W_{t}, & \text { in } \Omega \times[0,2 \pi] \times(0, T] ; \\ u(x, 0)=u_{0}(x), & x \in[0,2 \pi] .\end{cases}
$$

If $b=1$, then (7.2) is a degenerate linear SPDE satisfying $2 \alpha=C_{4}^{2}$. The exact solution is

$$
u(\omega, x, t)=u_{0}\left(x+W_{t}(\omega)\right) .
$$

In Table 3, we show the $L^{2}$-errors for the linear equation (7.2) with $b=1, M=100$ realizations and smooth initial condition $u_{0}(x)=\sin (x)$. For different terminal time $T$, the scheme has the expected $(k+1)$-th order of accuracy. 
TABLE 2. Verification of optimal convergence for nondegenerate linear SPDEs (7.1) with multiplicative noise: $k=2, M=1000$.

\begin{tabular}{llllllll}
\hline \hline & & \multicolumn{2}{c}{$b=0.1$} & \multicolumn{2}{c}{$b=0.5$} & \multicolumn{2}{c}{$b=1.0$} \\
\cline { 2 - 8 }$N=0.1$ & $N$ & $L^{2}$ Error & Order & $L^{2}$ Error & Order & $L^{2}$ Error & Order \\
\hline \multirow{5}{*}{$T=0$} & $1.94 \mathrm{E}-03$ & - & $1.96 \mathrm{E}-03$ & - & $2.03 \mathrm{E}-03$ & - \\
& 10 & $2.43 \mathrm{E}-04$ & 3.00 & $2.47 \mathrm{E}-04$ & 2.98 & $2.58 \mathrm{E}-04$ & 2.97 \\
& 40 & $3.03 \mathrm{E}-05$ & 3.00 & $3.07 \mathrm{E}-05$ & 3.01 & $3.20 \mathrm{E}-05$ & 3.01 \\
& 80 & $3.80 \mathrm{E}-06$ & 3.00 & $3.85 \mathrm{E}-06$ & 3.00 & $4.02 \mathrm{E}-06$ & 2.99 \\
& 160 & $4.75 \mathrm{E}-07$ & 3.00 & $4.83 \mathrm{E}-07$ & 2.99 & $5.05 \mathrm{E}-07$ & 2.99 \\
\hline \multirow{5}{*}{$T=0.5$} & 10 & $1.30 \mathrm{E}-03$ & - & $1.38 \mathrm{E}-03$ & - & $1.67 \mathrm{E}-03$ & - \\
& 20 & $1.64 \mathrm{E}-04$ & 2.99 & $1.75 \mathrm{E}-04$ & 2.97 & $2.12 \mathrm{E}-04$ & 2.97 \\
& 40 & $2.04 \mathrm{E}-05$ & 3.01 & $2.17 \mathrm{E}-05$ & 3.01 & $2.64 \mathrm{E}-05$ & 3.01 \\
& 80 & $2.55 \mathrm{E}-06$ & 3.00 & $2.73 \mathrm{E}-06$ & 2.99 & $3.41 \mathrm{E}-06$ & 2.95 \\
& 160 & $3.19 \mathrm{E}-07$ & 3.00 & $3.43 \mathrm{E}-07$ & 2.99 & $4.18 \mathrm{E}-07$ & 3.03 \\
\hline & 10 & $7.91 \mathrm{E}-04$ & - & $8.90 \mathrm{E}-04$ & - & $1.32 \mathrm{E}-03$ & - \\
& 20 & $9.96 \mathrm{E}-05$ & 2.99 & $1.14 \mathrm{E}-04$ & 2.97 & $1.71 \mathrm{E}-04$ & 2.95 \\
& 40 & $1.24 \mathrm{E}-05$ & 3.01 & $1.41 \mathrm{E}-05$ & 3.01 & $2.09 \mathrm{E}-05$ & 3.03 \\
& 80 & $1.55 \mathrm{E}-06$ & 3.00 & $1.79 \mathrm{E}-06$ & 2.98 & $2.83 \mathrm{E}-06$ & 2.88 \\
& 160 & $1.94 \mathrm{E}-07$ & 3.00 & $2.23 \mathrm{E}-07$ & 3.00 & $3.26 \mathrm{E}-07$ & 3.12 \\
\hline
\end{tabular}

TABLE 3. Verification of optimal convergence for degenerate linear SPDEs (7.2) with smooth initial value: $b=1, M=100, u_{0}(x)=\sin (x)$.

\begin{tabular}{llllll}
\hline \hline & & \multicolumn{2}{c}{$k=1$} & \multicolumn{2}{c}{$k=2$} \\
\cline { 3 - 6 }$N=0.1$ & $N$ & $L^{2}$ Error & Order & $L^{2}$ Error & Order \\
\hline \multirow{5}{*}{$T=0$} & 10 & $4.27 \mathrm{E}-02$ & - & $2.29 \mathrm{E}-03$ & - \\
& 20 & $1.07 \mathrm{E}-02$ & 2.00 & $2.73 \mathrm{E}-04$ & 3.07 \\
& 40 & $2.66 \mathrm{E}-03$ & 2.00 & $3.36 \mathrm{E}-05$ & 3.02 \\
& 80 & $6.65 \mathrm{E}-04$ & 2.00 & $4.20 \mathrm{E}-06$ & 3.00 \\
& 160 & $1.66 \mathrm{E}-04$ & 2.00 & $5.24 \mathrm{E}-07$ & 3.00 \\
\hline \multirow{5}{*}{$T=0.5$} & 10 & $4.32 \mathrm{E}-02$ & - & $2.27 \mathrm{E}-03$ & - \\
& 20 & $1.07 \mathrm{E}-02$ & 2.02 & $2.74 \mathrm{E}-04$ & 3.05 \\
& 40 & $2.67 \mathrm{E}-03$ & 2.00 & $3.39 \mathrm{E}-05$ & 3.01 \\
& 80 & $6.66 \mathrm{E}-04$ & 2.00 & $4.19 \mathrm{E}-06$ & 3.02 \\
& 160 & $1.66 \mathrm{E}-04$ & 2.00 & $5.24 \mathrm{E}-07$ & 3.00 \\
\hline & 10 & $4.43 \mathrm{E}-02$ & - & $2.23 \mathrm{E}-03$ & - \\
& 20 & $1.07 \mathrm{E}-02$ & 2.05 & $2.75 \mathrm{E}-04$ & 3.02 \\
& 40 & $2.67 \mathrm{E}-03$ & 2.01 & $3.43 \mathrm{E}-05$ & 3.00 \\
& 80 & $6.66 \mathrm{E}-04$ & 2.00 & $4.20 \mathrm{E}-06$ & 3.03 \\
& 160 & $1.66 \mathrm{E}-04$ & 2.00 & $5.24 \mathrm{E}-07$ & 3.00 \\
\hline
\end{tabular}

We also consider the case that the initial condition is discontinuous

$$
u_{0}(x)= \begin{cases}1, & \text { if } \frac{\pi}{2} \leq x \leq \frac{3 \pi}{2} \\ 0, & \text { if } 0 \leq x<\frac{\pi}{2} \text { or } \frac{3 \pi}{2}<x \leq 2 \pi\end{cases}
$$

For this discontinuous case, we compute the solution up to $T=1.0$ with only one realization $M=1$. The results are shown in Figure 1. We observe that the scheme converges to the true solution when $N$ increases. There are oscillations arising near the discontinuities of the solution. 

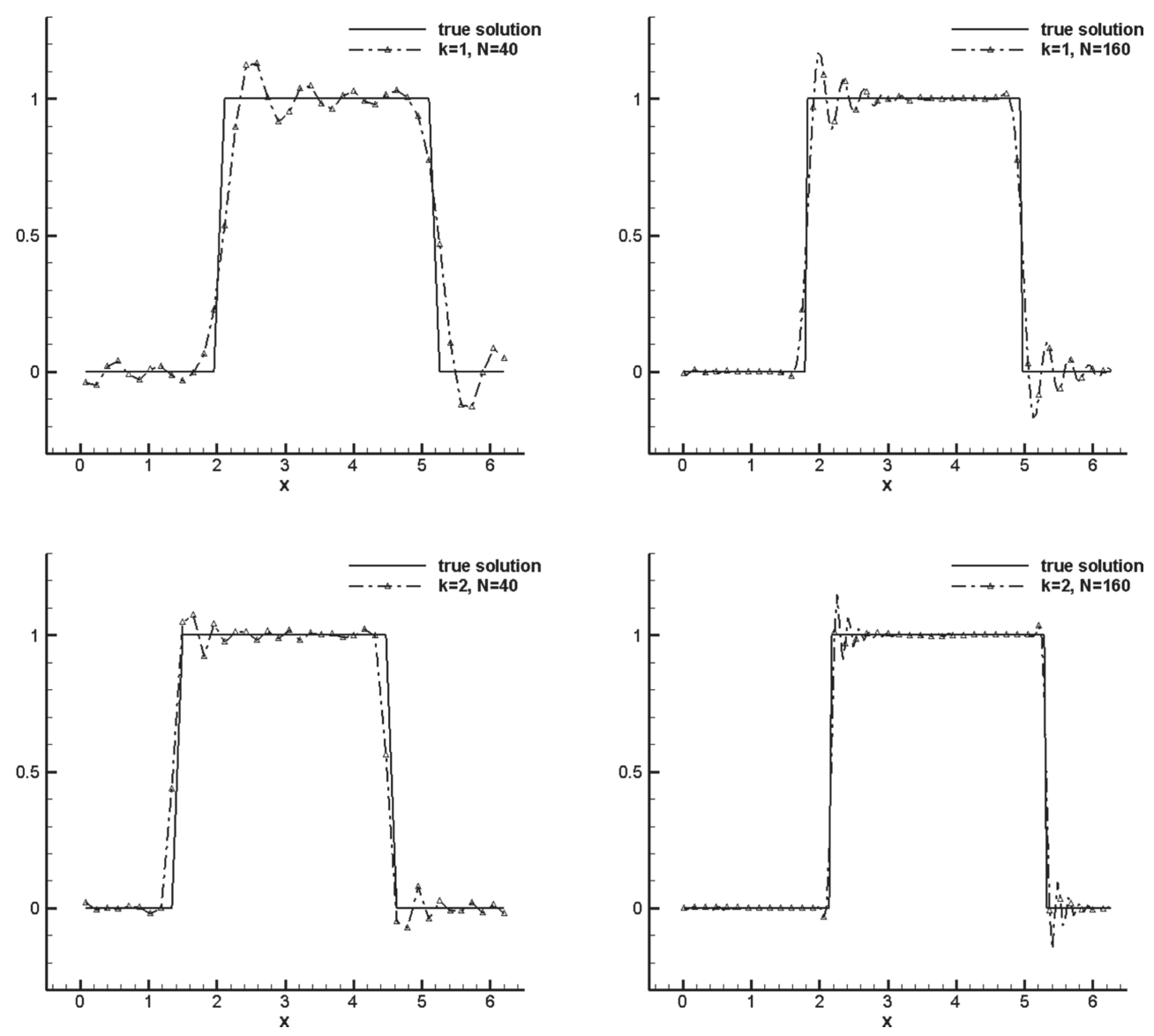

FIgURE 1. Performance of LDG scheme for degenerate linear SPDEs (7.2) with discontinuous initial value (7.3) and one fixed path: $b=1, M=1, T=1$.

Remark 7.1. If we set $b>1$ in our codes, i.e., the condition $2 \alpha-C_{4}^{2} \geq 0$ in Theorems 4.1 and 4.2 is not satisfied, then we find that the $L^{2}$-norm of the numerical solutions would explode, which confirms the necessity of the stochastic parabolicity condition.

Remark 7.2. The $L^{2}$-stability is very helpful in this discontinuous case, but is not enough to control the spurious numerical oscillations near the discontinuous region. In practice, it is worth trying to use limiters to control oscillations for the problems containing strong discontinuities, which will be investigated in the future.

\subsection{Stochastic viscous Burgers equation}

Although we cannot give error estimates for fully nonlinear equations, it is worth trying to apply the LDG method to solve some nonlinear equations. The next example is the stochastic viscous Burgers equation,

$$
\begin{cases}\mathrm{d} u=\left[\frac{\sigma^{2}}{2} u_{x x}-\frac{1}{2}\left(u^{2}\right)_{x}\right] \mathrm{d} t+\left(\sigma u_{x}+b\right) \mathrm{d} W_{t}, & \text { in } \Omega \times[0,2 \pi] \times(0, T] \\ u(x, 0)=\sin (x), & x \in[0,2 \pi] .\end{cases}
$$


TABLE 4. Verification of optimal convergence for stochastic viscous Burgers equation (7.4) with linear multiplicative noise: $b=\sigma=1, M=100$.

\begin{tabular}{llllll}
\hline \hline \multirow{5}{*}{$T=0.1$} & \multicolumn{3}{c}{$k=1$} & \multicolumn{2}{c}{$k=2$} \\
\cline { 3 - 6 } & $N$ & $L^{2}$ Error & Order & $L^{2}$ Error & Order \\
\hline \multirow{5}{*}{$T=0.4$} & $4.21 \mathrm{E}-02$ & - & $2.40 \mathrm{E}-03$ & - \\
& 20 & $1.07 \mathrm{E}-02$ & 1.97 & $2.90 \mathrm{E}-04$ & 3.05 \\
& 40 & $2.65 \mathrm{E}-03$ & 2.02 & $3.62 \mathrm{E}-05$ & 3.00 \\
& 80 & $6.69 \mathrm{E}-04$ & 1.99 & $4.54 \mathrm{E}-06$ & 3.00 \\
& 160 & $1.69 \mathrm{E}-04$ & 1.99 & $5.65 \mathrm{E}-07$ & 3.01 \\
\hline \multirow{5}{*}{$T=0.8$} & 10 & $5.87 \mathrm{E}-02$ & - & $5.83 \mathrm{E}-03$ & - \\
& 20 & $1.46 \mathrm{E}-02$ & 2.01 & $8.48 \mathrm{E}-04$ & 2.78 \\
& 40 & $3.64 \mathrm{E}-03$ & 2.00 & $1.01 \mathrm{E}-04$ & 3.07 \\
& 80 & $9.10 \mathrm{E}-04$ & 2.00 & $1.22 \mathrm{E}-05$ & 3.06 \\
& 160 & $2.29 \mathrm{E}-04$ & 1.99 & $1.51 \mathrm{E}-06$ & 3.02 \\
\hline & 10 & $2.21 \mathrm{E}-01$ & - & $7.98 \mathrm{E}-02$ & - \\
& 40 & $9.94 \mathrm{E}-02$ & 1.15 & $2.57 \mathrm{E}-02$ & 1.63 \\
& 80 & $3.74 \mathrm{E}-02$ & 1.41 & $5.97 \mathrm{E}-03$ & 2.11 \\
& 160 & $2.14 \mathrm{E}-02$ & 1.71 & $1.05 \mathrm{E}-03$ & 2.50 \\
\hline \multirow{5}{*}{1.2} & 10 & $5.58 \mathrm{E}-01$ & 2.01 & $9.00 \mathrm{E}-05$ & 3.55 \\
\hline & 20 & $4.31 \mathrm{E}-01$ & 0.37 & $3.16 \mathrm{E}-01$ & 0.35 \\
& 40 & $3.37 \mathrm{E}-01$ & 0.36 & $2.38 \mathrm{E}-01$ & 0.41 \\
& 80 & $2.62 \mathrm{E}-01$ & 0.36 & $1.91 \mathrm{E}-01$ & 0.32 \\
& 160 & $2.20 \mathrm{E}-01$ & 0.25 & $1.56 \mathrm{E}-01$ & 0.29 \\
\hline & & & & &
\end{tabular}

The exact solution of (7.4) is

$$
u(\omega, x, t)=v\left(x-b \int_{0}^{t} W_{s} \mathrm{~d} s+\sigma W_{t}, t\right)+b W_{t}
$$

where $v$ is the solution of the following deterministic inviscid Burgers equation

$$
\left\{\begin{aligned}
d v+\frac{1}{2}\left(v^{2}\right)_{x} \mathrm{~d} t & =0 & & \text { in }[0,2 \pi] \times(0, T), \\
v(x, 0) & =\sin (x), & & x \in[0,2 \pi] .
\end{aligned}\right.
$$

Note that the solution of (7.5) has an infinite slope - the wave "breaks" and a shock forms at

$$
T_{b}=\frac{-1}{\min v_{0}^{\prime}(x)}=1 .
$$

See [29]. So the exact solution of the stochastic viscous Burgers equation (7.4) also has a shock at $T_{b}=1$.

We use the simple Lax-Friedrichs flux for the nonlinear convection term $f(u)=\frac{u^{2}}{2}$,

$$
\widehat{f}\left(u^{-}, u^{+}\right)=\frac{1}{4}\left\{\left(u^{-}\right)^{2}+\left(u^{+}\right)^{2}\right\}-\frac{1}{2} \alpha\left(u^{+}-u^{-}\right)
$$

where

$$
\alpha=\max _{j}\left\{\left|u_{j+\frac{1}{2}}^{-}\right|,\left|u_{j+\frac{1}{2}}^{+}\right|\right\} .
$$

In Table 4, we show the $L^{2}$-errors for equation (7.4) with $b=\sigma=1$ and $M=100$ realizations. We see that the order of accuracy converges to $k+1$ when $T<T_{b}$. The scheme loses the order of accuracy as $T=1.2$ since the problem involves a shock when $T>T_{b}$. 

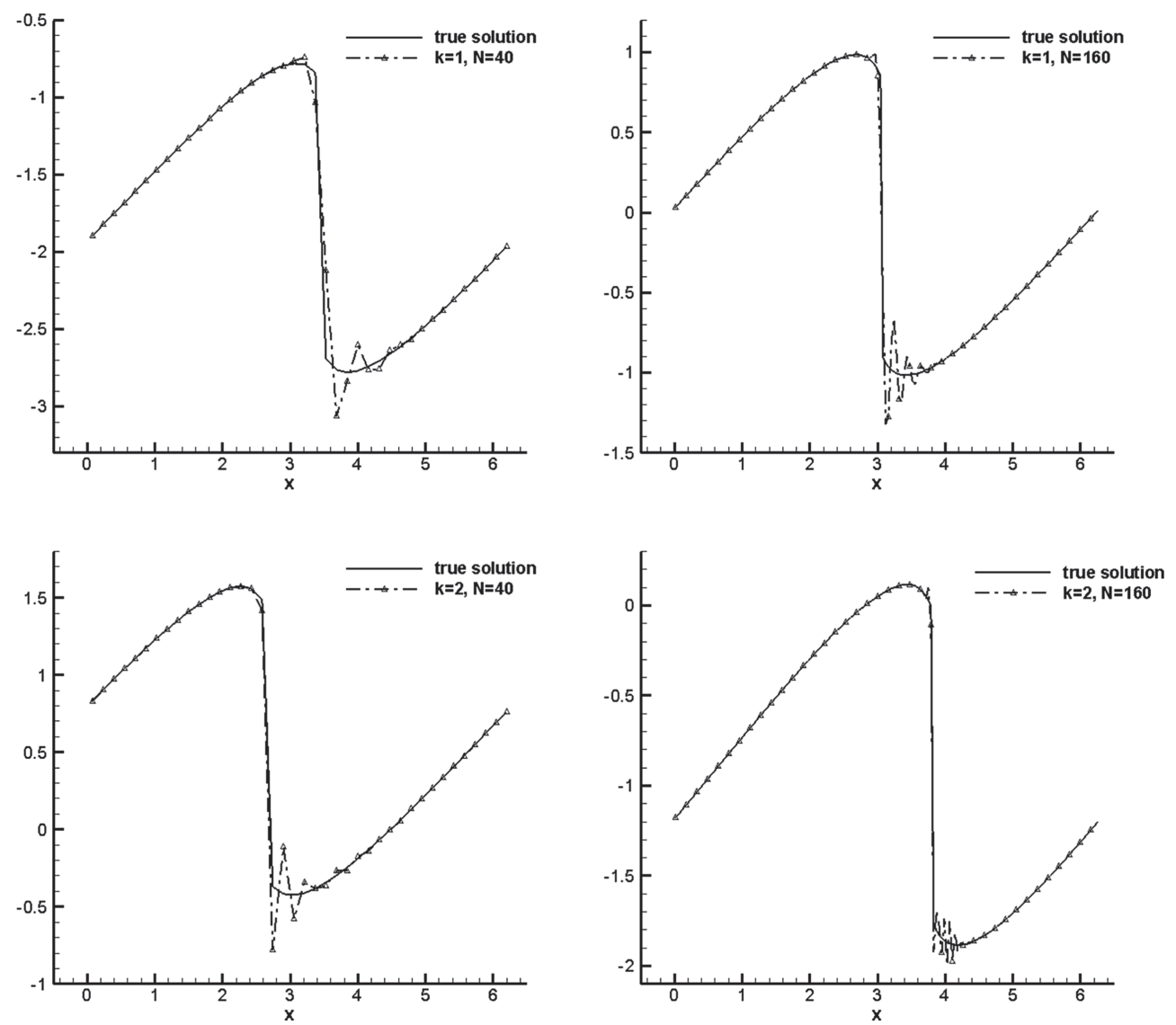

FiguRE 2. Performance of LDG scheme for stochastic viscous Burgers equation (7.4) with linear multiplicative noise and one fixed path: $b=1, \sigma=1, M=1, T=1.2$.

To see the behavior of numerical solution with $T>T_{b}$, we plot the approximate solution and the true solution at $T=1.2$ with $b=\sigma=1$ and only one realization $M=1$ to get Figure 2. We observe that the LDG scheme converges nicely to the exact solution for fixed stochastic path $\omega$. Again, some oscillations appear near the discontinuous region.

\subsection{Numerical test for studying the necessity of the stochastic parabolicity condition $2 \alpha \geq C_{4}^{2}$ for the LDG scheme}

Next we examine our numerical scheme on the following nonlinear SPDE to investigate the necessity of the stochastic parabolicity condition

$$
\begin{cases}\mathrm{d} u=\left[\left(u^{2} u_{x}\right)_{x}-u_{x}+3 u^{3}-\left(2+\frac{b^{2}}{2}\right) u\right] \mathrm{d} t+b u_{x} \mathrm{~d} W_{t}, & \text { in } \Omega \times[0,2 \pi] \times(0, T] \\ u(x, 0)=\sin (x), & x \in[0,2 \pi]\end{cases}
$$

One of the exact solutions of $(7.6)$ is

$$
u(\omega, x, t)=\sin \left(x+b W_{t}-t\right)
$$


TABLE 5. Verification of stability and convergence for nonlinear SPDEs (7.6) with multiplicative noise: $k=1, M=100$.

\begin{tabular}{llllllll}
\hline \hline & & \multicolumn{2}{c}{$b=0.05$} & \multicolumn{2}{c}{$b=0.1$} & \multicolumn{2}{c}{$b=0.5$} \\
\cline { 2 - 8 } & $N$ & $L^{2}$ Error & Order & $L^{2}$ Error & Order & $L^{2}$ Error & Order \\
\hline \multirow{5}{*}{$T=0.01$} & 10 & $3.17 \mathrm{E}-02$ & - & $3.17 \mathrm{E}-02$ & - & $3.25 \mathrm{E}-02$ & - \\
& 20 & $1.04 \mathrm{E}-02$ & 1.61 & $1.04 \mathrm{E}-02$ & 1.61 & $1.06 \mathrm{E}-02$ & 1.62 \\
& 40 & $2.73 \mathrm{E}-03$ & 1.92 & $2.74 \mathrm{E}-03$ & 1.92 & $3.95 \mathrm{E}-03$ & 1.42 \\
& 80 & $7.02 \mathrm{E}-04$ & 1.96 & $7.11 \mathrm{E}-04$ & 1.94 & $1.43 \mathrm{E}-03$ & 1.47 \\
& 160 & $1.77 \mathrm{E}-04$ & 1.99 & $1.78 \mathrm{E}-04$ & 2.00 & $1.69 \mathrm{E}-04$ & 3.08 \\
\hline \multirow{5}{*}{$T=0.1$} & 10 & $4.83 \mathrm{E}-02$ & - & $4.83 \mathrm{E}-02$ & - & $4.94 \mathrm{E}-02$ & - \\
& 20 & $1.21 \mathrm{E}-02$ & 2.00 & $1.22 \mathrm{E}-02$ & 1.99 & $1.41 \mathrm{E}-02$ & 1.81 \\
& 40 & $3.03 \mathrm{E}-03$ & 2.00 & $3.11 \mathrm{E}-03$ & 1.97 & $1.03 \mathrm{E}-02$ & 0.45 \\
& 80 & $7.54 \mathrm{E}-04$ & 2.01 & $8.01 \mathrm{E}-04$ & 1.96 & $1.01 \mathrm{E}-02$ & 0.03 \\
& 160 & $1.82 \mathrm{E}-04$ & 2.05 & $1.84 \mathrm{E}-04$ & 2.12 & $1.79 \mathrm{E}-01$ & -4.15 \\
\hline \multirow{5}{*}{$T=0.5$} & 10 & $5.52 \mathrm{E}-02$ & - & $5.57 \mathrm{E}-02$ & - & $6.44 \mathrm{E}-02$ & - \\
& 20 & $1.27 \mathrm{E}-02$ & 2.11 & $1.29 \mathrm{E}-02$ & 2.11 & $1.79 \mathrm{E}-02$ & 1.85 \\
& 40 & $3.15 \mathrm{E}-03$ & 2.02 & $3.22 \mathrm{E}-03$ & 2.00 & $1.62 \mathrm{E}-02$ & 0.15 \\
& 80 & $7.84 \mathrm{E}-04$ & 2.00 & $8.33 \mathrm{E}-04$ & 1.95 & $2.36 \mathrm{E}-01$ & -3.87 \\
& 160 & $1.94 \mathrm{E}-04$ & 2.01 & $2.51 \mathrm{E}-04$ & 1.73 & $5.92 \mathrm{E}-01$ & -1.33 \\
\hline
\end{tabular}

TABLE 6. Verification of stability and convergence for nonlinear SPDEs (7.6) with multiplicative noise: $k=2, M=100$.

\begin{tabular}{llllllll}
\hline \hline & & \multicolumn{2}{c}{$b=0.05$} & \multicolumn{2}{c}{$b=0.1$} & \multicolumn{2}{c}{$b=0.5$} \\
\cline { 2 - 8 }$T=0.01$ & $N$ & $L^{2}$ Error & Order & $L^{2}$ Error & Order & $L^{2}$ Error & Order \\
\hline \multirow{5}{*}{$T=0$} & $1.78 \mathrm{E}-03$ & - & $1.78 \mathrm{E}-03$ & - & $1.88 \mathrm{E}-03$ & - \\
& 10 & $2.67 \mathrm{E}-04$ & 2.74 & $2.68 \mathrm{E}-04$ & 2.74 & $3.08 \mathrm{E}-04$ & 2.61 \\
& 40 & $3.65 \mathrm{E}-05$ & 2.87 & $3.67 \mathrm{E}-05$ & 2.87 & $1.02 \mathrm{E}-04$ & 1.60 \\
& 80 & $4.57 \mathrm{E}-06$ & 3.00 & $4.67 \mathrm{E}-06$ & 2.97 & $2.71 \mathrm{E}-03$ & -4.73 \\
& 160 & $5.57 \mathrm{E}-07$ & 3.04 & $5.91 \mathrm{E}-07$ & 2.98 & $2.51 \mathrm{E}-01$ & -6.53 \\
\hline \multirow{5}{*}{$T=0.1$} & 10 & $4.70 \mathrm{E}-03$ & - & $4.71 \mathrm{E}-03$ & - & $5.33 \mathrm{E}-03$ & - \\
& 20 & $4.46 \mathrm{E}-04$ & 3.39 & $4.52 \mathrm{E}-04$ & 3.38 & $1.13 \mathrm{E}-03$ & 2.23 \\
& 40 & $4.44 \mathrm{E}-05$ & 3.33 & $4.50 \mathrm{E}-05$ & 3.33 & $2.38 \mathrm{E}-02$ & -4.39 \\
& 80 & $4.84 \mathrm{E}-06$ & 3.20 & $5.03 \mathrm{E}-06$ & 3.16 & $4.62 \mathrm{E}-01$ & -4.28 \\
& 160 & $5.61 \mathrm{E}-07$ & 3.11 & $2.48 \mathrm{E}-06$ & 1.02 & $6.09 \mathrm{E}-01$ & -0.40 \\
\hline & 10 & $4.18 \mathrm{E}-03$ & - & $4.30 \mathrm{E}-03$ & - & $8.15 \mathrm{E}-03$ & - \\
& 20 & $4.35 \mathrm{E}-04$ & 3.26 & $4.60 \mathrm{E}-04$ & 3.23 & $2.15 \mathrm{E}-02$ & -1.40 \\
& 40 & $4.62 \mathrm{E}-05$ & 3.24 & $4.73 \mathrm{E}-05$ & 3.28 & $5.43 \mathrm{E}-01$ & -4.66 \\
& 80 & $4.89 \mathrm{E}-06$ & 3.24 & $5.70 \mathrm{E}-06$ & 3.05 & NaN & NaN \\
& 160 & $5.72 \mathrm{E}-07$ & 3.10 & $3.53 \mathrm{E}-03$ & -9.27 & $6.97 \mathrm{E}-01$ & NaN \\
\hline
\end{tabular}

Notice that in this case $\alpha$ is equal to 0 and $|b|$ is greater than 0 , so the stochastic parabolic condition $2 \alpha \geq C_{4}^{2}$ in Theorems 4.1 and 4.2 is not satisfied, and instability appears for our scheme in this numerical test. Tables 5 and 6 display the $L^{2}$-errors for the nonlinear equation (7.6) with $M=100$ realizations. We see that the LDG scheme works well when the terminal time $T$ and the stochastic coefficient $b$ are small, in which the order of accuracy is $k+1$. When $T$ and $b$ are large enough, similar to the situation we described in Remark 7.1, we lose the order of accuracy and the scheme seems unstable, which again suggests the necessity of the stochastic parabolicity condition $2 \alpha \geq C_{4}^{2}$. 
TABLE 7. Verification of optimal convergence for nondegenerate nonlinear SPDEs (7.8) with multiplicative noise: $b=1, M=100$.

\begin{tabular}{llllll}
\hline \hline & & \multicolumn{2}{c}{$k=1$} & \multicolumn{2}{c}{$k=2$} \\
\cline { 3 - 6 }$T$ & $N$ & $L^{2}$ Error & Order & $L^{2}$ Error & Order \\
\hline \multirow{5}{*}{$T=0.1$} & 10 & $4.37 \mathrm{E}-02$ & - & $2.18 \mathrm{E}-03$ & - \\
& 20 & $1.07 \mathrm{E}-02$ & 2.03 & $2.69 \mathrm{E}-04$ & 3.02 \\
& 40 & $2.67 \mathrm{E}-03$ & 2.01 & $3.36 \mathrm{E}-05$ & 3.00 \\
& 80 & $6.66 \mathrm{E}-04$ & 2.00 & $4.19 \mathrm{E}-06$ & 3.00 \\
& 160 & $1.66 \mathrm{E}-04$ & 2.00 & $5.24 \mathrm{E}-07$ & 3.00 \\
\hline \multirow{5}{*}{$T=0.5$} & 10 & $4.75 \mathrm{E}-02$ & - & $2.19 \mathrm{E}-03$ & - \\
& 20 & $1.09 \mathrm{E}-02$ & 2.12 & $2.70 \mathrm{E}-04$ & 3.02 \\
& 40 & $2.68 \mathrm{E}-03$ & 2.03 & $3.36 \mathrm{E}-05$ & 3.01 \\
& 80 & $6.67 \mathrm{E}-04$ & 2.01 & $4.19 \mathrm{E}-06$ & 3.00 \\
& 160 & $1.66 \mathrm{E}-04$ & 2.00 & $5.24 \mathrm{E}-07$ & 3.00 \\
\hline & 10 & $1.74 \mathrm{E}-01$ & - & $2.42 \mathrm{E}-03$ & - \\
& 20 & $1.79 \mathrm{E}-02$ & 3.28 & $2.87 \mathrm{E}-04$ & 3.08 \\
& 40 & $3.30 \mathrm{E}-03$ & 2.44 & $3.62 \mathrm{E}-05$ & 2.99 \\
& 80 & $7.17 \mathrm{E}-04$ & 2.21 & $4.45 \mathrm{E}-06$ & 3.02 \\
& 160 & $1.70 \mathrm{E}-04$ & 2.08 & $5.56 \mathrm{E}-07$ & 3.00 \\
\hline
\end{tabular}

\subsection{Nondegenerate nonlinear SPDEs}

Note that (7.7) is also the exact solution of the following nondegenerate nonlinear equation,

$$
\begin{cases}\mathrm{d} u=\left[\left(u^{2} u_{x}\right)_{x}+\left(2+\frac{b^{2}}{2}\right) u_{x x}-u_{x}+3 u^{3}\right] \mathrm{d} t+b u_{x} \mathrm{~d} W_{t} & \text { in } \Omega \times[0,2 \pi] \times(0, T] ; \\ u(x, 0)=\sin (x), & x \in[0,2 \pi] .\end{cases}
$$

We see that in this case the constant $\alpha$ equals to $2+\frac{b^{2}}{2}$, which implies that the condition $\alpha-\frac{b^{2}}{2}=2>0$ is satisfied.

In Table 7, we show the $L^{2}$-errors for the equation (7.8) with $b=1$ and $M=100$ realizations, which indicates that the LDG method gives the expected $(k+1)$-th order of accuracy for the nondegenerate nonlinear problems.

\section{CONCLUding REMARKS}

In this article, we present a semi-discrete LDG scheme for fully nonlinear parabolic SPDEs. The $L^{2}$-stability results of the scheme are obtained, and the optimal error estimates of order $\mathcal{O}\left(h^{k+1}\right)$ for semilinear stochastic equations are proved. We combine an explicit derivative-free order 1.5 time discretization scheme to perform several numerical experiments on some model problems to confirm the analytical results.

Acknowledgements. The work of the first and the third authors was supported by National Key R\&D Program of China (Grant No. 2018YFA0703900) and National Natural Science Foundation of China (Grant No. 11631004). The work of the second author was supported by ARO grant W911NF-16-1-0103 and NSF grant DMS-1719410. The authors would like to thank both reviewers and the editor for their helpful comments. The first author would also like to thank Professor Kai Du for his advice on the theory of SPDEs.

\section{REFERENCES}

[1] I. Babuska, R. Tempone and G.E. Zouraris, Galerkin finite element approximations of stochastic elliptic partial differential equations. SIAM J. Numer. Anal. 42 (2004) 800-825. 
[2] F. Bassi and S. Rebay, A high-order accurate discontinuous finite element method for the numerical solution of the compressible Navier-Stokes equations. J. Comput. Phys. 131 (1997) 267-279.

[3] A. Bensoussan, Some existence results for stochastic partial differential equations. In: Stochastic Partial Differential Equations and Applications (Trento, 1990). Longman Sci. Tech., Harlow (1992) 37-53.

[4] Y. Cao, R. Zhang and K. Zhang, Finite element and discontinuous Galerkin method for stochastic Helmholtz equation in twoand three-dimensions. J. Comput. Math. 26 (2008) 702-715.

[5] Y. Cao, R. Zhang and K. Zhang, Finite element method and discontinuous Galerkin method for stochastic scattering problem of Helmholtz type in $\mathbb{R}^{d}(d=2,3)$. Potential Anal. 28 (2008) 301-319.

[6] P. Castillo, B. Cockburn, D. Schotzau and C. Schwab, Optimal a priori error estimates for the $h p$-version of the local discontinuous Galerkin method for convection-diffusion problems. Math. Comput. 71 (2002) 455-478.

[7] T. Chen, B. Rozovskii and C.-W. Shu, Numerical solutions of stochastic PDEs driven by arbitrary type of noise. Stoch. Part. Differ. Equ.: Anal. Comput. 7 (2019) 1-39.

[8] P. Ciarlet, The Finite Element Method for Elliptic Problem. North Holland (1975).

[9] B. Cockburn and C.-W. Shu, The Runge-Kutta local projection P1-discontinuous Galerkin method for scalar conservation laws. MMNP 25 (1991) 337-361.

[10] B. Cockburn and C.-W. Shu, TVB Runge-Kutta local projection discontinuous Galerkin finite element method for conservation laws II: general framework. Math. Comput. 52 (1998) 411-435.

[11] B. Cockburn and C.-W. Shu, The Runge-Kutta discontinuous Galerkin method for conservation laws V: multidimensional systems. J. Comput. Phys. 141 (1998) 199-224.

[12] B. Cockburn and C.-W. Shu, The local discontinuous Galerkin method for time-dependent convection-diffusion systems. SIAM J. Numer. Anal. 35 (1998) 2440-2463.

[13] B. Cockburn, S.-Y. Lin and C.-W. Shu, TVB Runge-Kutta local projection discontinuous Galerkin finite element method for conservation laws III: one dimensional systems. J. Comput. Phys. 84 (1989) 90-113.

[14] B. Cockburn, S. Hou and C.-W. Shu, The Runge-Kutta local projection discontinuous Galerkin finite element method for conservation laws IV: the multidimensional case. Math. Comput. 54 (1990) 545-581.

[15] Y.L. Dalecky and N.Y. Goncharuk, On a quasilinear stochastic differential equation of parabolic type. Stoch. Anal. App. 12 (1994) 103-129.

[16] A.M. Davie and J.G. Gaines, Convergence of numerical schemes for the solution of parabolic stochastic partial differential equations. Math. Comput. 70 (2001) 121-134.

[17] A. Debussche and J. Printems, Weak order for the discretization of the stochastic heat equation. Math. Comput. 78 (2009) $845-863$.

[18] K. Du and J. Liu, On the Cauchy problem for stochastic parabolic equations in Hölder spaces. Trans. Am. Math. Soc. 371 (2019) 2643-2664.

[19] Q. Du and T. Zhang, Numerical approximation of some linear stochastic partial differential equations driven by special additive noises. SIAM J. Numer. Anal. 40 (2002) 1421-1445.

[20] N.Y. Goncharuk, On a class of quasilinear stochastic differential equations of parabolic type: regular dependence of solutions on initial data. In: Stochastic Partial Differential Equations. Cambridge University Press, Cambridge (1995) 97-119.

[21] I. Gyöngy, Lattice approximations for stochastic quasi-linear parabolic partial differential equations driven by space-time white noise I. Potential Anal. 9 (1998) 1-25.

[22] I. Gyöngy and T. Martinez, On numerical solution of stochastic partial differential equations of elliptic type. Stochastics $\mathbf{7 8}$ (2006) 213-231.

[23] S. He, J. Wang and J. Yan, Semimartingale Theory and Stochastic Calculus. Science Press, New York (1992).

[24] M. Hofmanová, Strong solutions of semilinear stochastic partial differential equations. Nonlinear Differ. Equ. App. 20 (2013) $757-778$.

[25] A. Jentzen and P.E. Kloeden, Overcoming the order barrier in the numerical approximation of stochastic partial differential equations with additive space-time noise. Proc. R. Soc. A: Math. Phys. Eng. Sci. 465 (2009) 649-667.

[26] G. Jiang and C.-W. Shu, On a cell entropy inequality for discontinuous Galerkin methods. Math. Comput. 62 (1994) 531-538.

[27] P. Kloeden and E. Platen, Numerical Solution of Stochastic Differential Equations, 3rd ed. In: Vol. 23 of Applications in Mathematics, Stochastic Modelling and Applied Probability. Springer-Verlag, Berlin (1999).

[28] M. Kovacs, S. Larsson and F. Saedpanah, Finite element approximation of the linear stochastic wave equation with additive noise. SIAM J. Numer. Anal. 48 (2010) 408-427.

[29] R.J. LeVeque, Numerical Methods for Conservation Laws. In: Lectures in Mathematics. Birkhauser, Basel (1992).

[30] Y. Li, C.-W. Shu and S. Tang, A discontinuous Galerkin method for stochastic conservation laws. SIAM J. Sci. Comput. 42 (2020) A54-A86.

[31] G.J. Lord and T. Shardlow, Postprocessing for stochastic parabolic partial differential equations. SIAM J. Numer. Anal. 45 (2007) 870-889.

[32] X. Mao, Stochastic Differential Equations and Applications, 2nd ed. Horwood, Chichester (2008).

[33] A. Millet and P.L. Morien, On implicit and explicit discretization schemes for parabolic SPDEs in any dimension. Stoch. Process. App. 115 (2005) 1073-1106.

[34] T. Müller-Gronbach and K. Ritter, Lower bounds and nonuniform time discretization for approximation of stochastic heat equations. Found. Comput. Math. 7 (2007) 135-181. 
[35] T. Müller-Gronbach, K. Ritter and T. Wagner, Optimal pointwise approximation of infinite-dimensional Ornstein-Uhlenbeck processes. Stoch. Dyn. 8 (2008) 519-541.

[36] É. Pardoux, Stochastic Partial Differential Equations. Lecture notes for the course given at Fudan University, Shanghai (2007).

[37] É. Pardoux and S. Peng, Backward doubly stochastic differential equations and systems of quasilinear SPDEs. Probab. Theory Relat. Fields 98 (1994) 209-227.

[38] W. Pazner, N. Trask and P.J. Atzberger, Stochastic discontinuous Galerkin methods (SDGM) based on fluctuation-dissipation balance. Results Appl. Math. 4 (2019) 100068.

[39] C. Prévôt and M. Röckner, A Concise Course on Stochastic Partial Differential Equations. In: Vol. 1905 of Lecture Notes in Mathematics. Springer, Berlin (2007).

[40] P. Protter, Stochastic Integration and Differential Equations, 2nd ed. Springer-Verlag, New York (2004).

[41] M. Roozbahani, H. Aminikhah and M. Tahmasebi, Numerical solution of nonlinear SPDEs using a multi-scale method. Comput. Methods Diff. Equ. 6 (2018) 157-175.

[42] C. Roth, A combination of finite difference and Wong-Zakai methods for hyperbolic stochastic partial differential equations. Stoch. Anal. App. 24 (2006) 221-240.

[43] P.E. Souganidis, Fully nonlinear first- and second-order stochastic partial differential equations. In: CIME Lecture Notes (2016) $1-37$.

[44] J.B. Walsh, Finite element methods for parabolic stochastic PDEs. Potential Anal. 23 (2005) 1-43.

[45] J.B. Walsh, On numerical solutions of the stochastic wave equation. Illinois J. Math. 50 (2006) 991-1018.

[46] H.-J. Wang and Q. Zhang, Error estimate on a fully discrete local discontinuous Galerkin method for linear convection-diffusion problem. J. Comput. Math. 31 (2013) 283-307.

[47] Y. Yan, Galerkin finite element methods for stochastic parabolic partial differential equations. SIAM J. Numer. Anal. 43 (2005) 1363-1384. 